\title{
Synthesis, properties, and biomedical applications of gelatin methacryloyl
}

\section{(GelMA) hydrogels}

Kan Yue ${ }^{1,2^{*}}$, Grissel Trujillo-de Santiago ${ }^{1,2,3^{*}}$, Mario Moisés Alvarez ${ }^{1,2,3}$, Ali Tamayol ${ }^{1,2}$, Nasim Annabi $^{1,2,4,5^{* *}}$, Ali Khademhosseini ${ }^{1,2,4,6^{* *}}$

(1) Biomaterials Innovation Research Center, Division of Biomedical Engineering, Department of Medicine, Brigham and Women's Hospital, Harvard Medical School, Boston 02139, MA, USA

(2) Harvard-Massachusetts Institute of Technology Division of Health Sciences and Technology, Massachusetts Institute of Technology, Cambridge 02139, MA, USA.

(3) Centro de Biotecnología-FEMSA, Tecnológico de Monterrey at Monterrey, Ave. Eugenio Garza Sada 2501 Sur Col. Tecnológico, CP 64849, Monterrey, Nuevo León, México.

(4) Wyss Institute for Biologically Inspired Engineering, Harvard University, Boston 02115, MA, USA

(5) Department of Chemical Engineering, Northeastern University, Boston, MA, 02115-5000, USA.

(6) Department of Physics, King Abdulaziz University, Jeddah 21569, Saudi Arabia.

*Kan Yue and Grissel Trujillo-de Santiago contributed equally to this work.

${ }^{* *}$ Correspondence and requests for materials should be addressed to Ali Khademhosseini (alik@rics.bwh.harvard.edu) and Nasim Annabi (‥annabi@neu.edu $)$ 
Abstract. Gelatin methacryloyl (GelMA) hydrogels have been widely used for various biomedical applications due to their suitable biological properties and tunable physical characteristics. Three dimensional (3D) GelMA hydrogels closely resemble some essential properties of native extracellular matrix $(\mathrm{ECM})$ due to the presence of cell-attaching and matrix metalloproteinase responsive peptide motifs, which allow cells to proliferate and spread in GelMA-based scaffolds. GelMA is also versatile from a processing perspective. It crosslinks when exposed to light irradiation to form hydrogels with tunable mechanical properties which mimic the native ECM. It can also be microfabricated using different methodologies including micromolding, photomasking, bioprinting, self-assembly, and microfluidic techniques to generate constructs with controlled architectures. Hybrid hydrogel systems can also be formed by mixing GelMA with nanoparticles such as carbon nanotubes and graphene oxide, and other polymers to form networks with desired combined properties and characteristics for specific biological applications. Recent research has demonstrated the proficiency of GelMA-based hydrogels in a wide range of applications including engineering of bone, cartilage, cardiac, and vascular tissues, among others. Other applications of GelMA hydrogels, besides tissue engineering, include fundamental single-single cell research, cell signaling, drug and gene delivery, and bio-sensing. 


\section{Introduction}

Hydrogels are crosslinked network of hydrophilic polymers that can swell in water to capture many times their original mass. Physical and biochemical properties of hydrogels largely depend on their compositions, methods used for their polymerization, and their crosslinking density. Hydrogels provide a versatile platform to include desired combinations of properties for designed applications [1]. Numerous hydrogels have been developed based on natural and/or synthetic polymers and various kinds of crosslinking chemistry towards different biomedical applications, such as regenerative medicine, drug delivery, and tissue adhesives [2]. In particular, hydrogels for biomedical applications are designed to resemble the characteristics of native extracellular matrix (ECM) and to provide three-dimensional (3D) supports for cellular growth and tissue formation [3]. Hydrogels have been also widely used in 3D culturing to study cell-matrix and cell-cell interactions, proliferation, migration [4], and controlled differentiation [5]. To this aim, hydrogels based on naturally occurring biopolymers have many advantages over synthetic polymers, such as excellent biocompatibility, low immunoresponse, and possible bioactive motifs encoded in their chemical structures.

In this contribution, we review recent research on the synthesis, characterizations and biomedical applications of gelatin methacryloyl (GelMA), which is also frequently referred as gelatin methacrylate [6-9], methacrylated gelatin [10-13], methacrylamide modified gelatin [14], or gelatin methacrylamide [15-18] in literature by different authors. Based on the fact that GelMA is a gelatin derivative containing a majority of methacrylamide groups and a 
minority of methacrylate groups, we suggest that gelatin methacryloyl is a more suitable name, which also matches the widely accepted abbreviation GelMA.

GelMA undergoes photoinitiated radical polymerization (i.e. under UV light exposure with the presence of a photoinitiator) to form covalently crosslinked hydrogels. As the hydrolysis product of collagen, the major component of ECM in most tissues, gelatin contains many arginine-glycine-aspartic acid (RGD) sequences that promote cell attachment [19], as well as the target sequences of matrix metalloproteinase (MMP) that are suitable for cell remodeling [20]. When compared to collagen, the advantages of gelatin include better solubility and less antigenicity [21, 22]. The hydrolysis process also denatures the tertiary structure of collagen, reducing its structural variations due to different sources. A gelatin solution has, on its own, the unique property of gelation at low temperatures to form physically crosslinked hydrogels $[14,23]$. In addition, several chemical reactions have been applied to covalently crosslink gelatin [24-27]. Conveniently, introduction of methacryloyl substituent groups confers to gelatin the property of photocrosslinking with the assistance of a photoinitiator and exposure to light, due to the photopolymerization of the methacryloyl substituents [14]. This polymerization can take place at mild conditions (room temperature, neutral $\mathrm{pH}$, in aqueous environments, etc.), and allows for temporal and spatial control of the reaction [6]. This enables microfabrication of the hydrogels to possess unique patterns, morphologies, and 3D structures, providing ideal platforms to control cellular behaviors, to study cell-biomaterial interactions, and to engineer tissues [6, 28].

It is worth mentioning that the chemical modification of gelatin by MA generally only 
involves less than 5\% of the amino acid residues in molar ratio [14], which implies that most of the functional amino acid motifs (such as the RGD motifs and MMP-degradable motifs) will not be significantly influenced. Specifically, the RGD motifs do not contain groups that will react with MA, which ensures the retention of good cell adhesive properties of the GelMA [6, 19, 29]. Furthermore, the in vitro enzymatic degradation of GelMA hydrogels by type I and type II collagenases (also known as MMP-1 and MMP-8, respectively) proceeds at accelerated rates, indicating the existence of MMP-sensitive motifs in GelMA [30, 31].

Since its first synthesis report [14], GelMA hydrogels have been thoroughly studied in terms of physical and biochemical properties for many different applications ranging from tissue engineering, to drug and gene delivery. In this review, we will focus on studies related to GelMA hydrogel synthesis and characterization as well as its composites, and summarize the reported methods for microfabrication of GelMA hydrogels, and the resulting applications of these GelMA-based biomaterials.

\section{Synthesis and characterization of GelMA hydrogels}

Different protocols have been reported for the preparation of methacryloyl-substituted gelatin, but they are all essentially minor variations of a general method first reported by Van Den Bulcke et al. [14]. Briefly, GelMA is synthesized by the direct reaction of gelatin with methacrylic anhydride (MA) in phosphate buffer at $50{ }^{\circ} \mathrm{C}$. This reaction introduces methacryloyl substitution groups on the reactive amine and hydroxyl groups of the amino acid residues [14] (Figure 1A). Typically, GelMA is synthesized by the direct reaction of gelatin with methacrylic anhydride (MA) in phosphate buffer $(\mathrm{pH}=7.4)$ at $50{ }^{\circ} \mathrm{C}$. Different 
degrees of methacryloyl substitution can be achieved in GelMA by tunning the amount of MA added to the reaction mixture, which produces GelMA with different mechanical properties. Maintaining a higher $\mathrm{pH}$ during the reaction enhances the reactivity of amine and hydroxyl groups, thereby leading to a higher degree of substitution [32]. After the substitution reaction is stopped by diluting the reaction mixture (typically $5 \mathrm{X}$ ) with phosphate buffer, the resulting solution should then be dialyzed against deionized water through a dialysis tubing (12-14 kDa molecular weight cutoff) for 5 to 7 days to allow complete removal of the low-molecular-weight impurities (including unreacted methacrylic anhydride and methacrylic acid byproducts, etc.), which are potentially cytotoxic. For best results, it is recommended to change the dialysis water at least twice a day. Finally, the dialyzed solution can be freeze dried and stored, preferably under refrigeration, until use. Note that the reaction of gelatin and MA is a two-phase reaction, where an organic compound is added and dispersed into an aqueous phase. As a result, the rate of MA addition and the conditions of mixing might have specific effects on the quality of the dispersion, and consequently, on the degree of methacryloyl substitution in the final product. To our knowledge, the effect of different mixing conditions on the properties of the resulting GelMA has not been studied in detail and remains a topic for further optimization studies.

Photocrosslinking of the product GelMA can be conducted using a water-soluble initiator under UV light. Common choices for water-soluble photoinitiators include 2-hydroxy-1-[4-(2-hydroxyethoxy)phenyl]-2-methyl-1-propanone (Irgacure 2959) [6, 29] and lithium acylphosphinate salt (LAP) [33]. Irgacure 2959, a commercially available 
photoinitiator, has a solubility in water of at least $5 \mathrm{mg} / \mathrm{mL}$ [34], which is sufficiently high for most photopolymerization conducted in aqueous environments. LAP, a recently developed alternative water-soluble photoinitiator, has a higher solubility in water (up to $8.5 \mathrm{wt} \%$ ) and a higher molar extinction coefficient at $365 \mathrm{~nm}$ than Irgacure 2959 [33]. The degree of substitution, GelMA concentration, initiator concentration, and UV exposure time are the major parameters that allow tuning of the physical properties of the resulting GelMA hydrogels [14].

Characterization of the physical properties (i.e. porosity, compressive modulus, and water swelling) and cell response parameters (i.e. cell viability, proliferation, and spreading) on GelMA hydrogels is key to determining the suitability of these polymers for different tissue engineering applications. GelMA offers much versatility regarding the tuning of its characteristics by manipulating its synthesis and processing (i.e. conditions of crosslinking). For example, the compressive modulus of GelMA can be fine-tuned by varying the degree of methacryloyl substitution or by adding inorganic or organic components to GelMA (Figure 1B), making it suitable for a wide range of biomedical applications. GelMA hydrogels can also be subjected to cryogenic treatments (i.e. freeze drying) to generate porous scaffolds with controlled pore sizes and porosity [35]. Van Vlierberghe et al. reported the preparation of porous GelMA hydrogels upon cryogenic treatments of the chemically crosslinked hydrogels [36]. They identified that the average pore sizes were inversely related to the concentration of GelMA solution and the cooling rate, and they successfully prepared GelMA hydrogels with gradient pore sizes using a gradient cooling rate strategy [36, 37]. We and others have also 
shown that the pore sizes in GelMA hydrogel can be tuned by changing the degree of methacryloyl substitution (Figure 1C) [9, 38]. For example, Chen et al. [9] synthetized GelMA hydrogels with different substitution degrees (49.8, 63.8, and 73.2\%) using 1,5 , and $10 \mathrm{M}$ methacrylic anhydrate solutions, respectively. In these experiments, the degree of substitution was defined as the ratio between functionalized to originally available amino groups, as measured by H-NMR spectroscopy. The average pore size of the resulting GelMA hydrogels, as characterized by SEM after freeze drying, was 50 (49.8\%), 30 (63.8\%), and $25 \mu \mathrm{m}(73.2 \%)$.

Other relationships have been reported between several characteristics (chemical, physical, and bio-responsive) of GelMA hydrogels. Chen et al. observed that the compressive modulus of a GelMA hydrogel was directly proportional to the degree of methacryloyl substitution $(2.0 \pm 0.18 \mathrm{KPa}(49.8 \%), 3.2 \pm 0.18 \mathrm{KPa}(63.8 \%)$, and $4.5 \pm 0.33 \mathrm{KPa}(73.2 \%))$ [9]. The compressive modulus was also directly proportional to the GelMA mass/volume fraction. For example, Nichol et al. estimated compressive modulus values of 2.0, 10.0, and $22.0 \mathrm{KPa}$, respectively, for 5, 10, and 15\% w/v GelMA (with a degree of substitution of 53.8\%). The corresponding values for GelMA with a higher degree of substitution (81.4\%) are higher: 3.3, 16.0, and $30.0 \mathrm{KPa}$ for 5, 10 and 15\% w/v GelMA [6]. Nichol et al. reported that the swelling ratio decreased as increasing the degree of methacryloyl substitution and the GelMA mass fraction [6]. Similarly, cell proliferation was inversely proportional to the GelMA mass fraction within the hydrogel [6].

The attachment and proliferation of different cell lines in GelMA hydrogels have been widely established and characterized. Pioneering studies demonstrated that GelMA hydrogels 
can serve as an active cell culture matrix material in both $2 \mathrm{D}$ and $3 \mathrm{D}$ experiments, due to the combined biocompatibility, mechanical properties, and existence of bio-active peptide sequences [39]. For instance, cells can be suspended in GelMA prepolymer solutions and crosslinked upon exposure to UV light to form cell-laden 3D hydrogels. High cell viability $(>80 \%)$ is generally observed in these photocrosslinked cell-laden GelMA hydrogels. In contrast to 2D cell culture, cells encapsulated in hydrogels should be able to remodel their surrounding environments for spreading and migration [29].

\section{Microfabrication of GelMA hydrogels}

The excellent biocompatibility of GelMA hydrogels makes them suitable as cell culture matrices that mimic native ECM. However, to fabricate tissue constructs similar to living tissues, one of the essential requirements is to generate organized assemblies of various types of cells to resemble the complex architectures of the targeting tissues in vitro. Recently, several state-of-the-art microfabrication techniques have been explored to control the 3D microstructure of GelMA hydrogels, and in return, to tune the cell-material interactions and thus, cell behaviors. In this section, we will briefly summarize the microengineered GelMA hydrogels produced by using several microscale techniques (Figure 2).

\subsection{Photopatterned GelMA hydrogels}

Photopatterning can be simply defined as a set of techniques that uses any form of light to imprint patterns into materials. Since GelMA is a photocrosslinkable material, the idea of using photopatterning techniques to impart a particular topography or to engineer 3D architectures of GelMA-based scaffolds is quite intuitive. Photopatterning of GelMA hydrogels with the 
assistance of photomasks is a convenient method to achieve micropatterned GelMA scaffolds. Light irradiation will only pass through the transparent regions of the photomask and induce chemical crosslinking of the GelMA solution underneath, resulting in hydrogels imprinted with the photomask patterns (Figure 2A). Complex architectures within GelMA hydrogels have been created by using photomasking techniques. For example, Nichol et al. utilized this method to fabricate GelMA microgels with square shapes [6]. 3T3 fibroblast cells encapsulated within these microgels showed similar viability $(75-92 \%)$ but more elongated morphologies than those in bulk GelMA hydrogels. Moreover, human umbilical vein endothelial cells (HUVECs) seeded on a patterned substrate coated with either GelMA hydrogel or polyethylene glycol (PEG) chains exhibited selective binding to GelMA surfaces. They also demonstrated the formation of a microchannel within a 3T3 cell-laden GelMA hydrogel, through which HUVECs can be perfused in to form a co-cultured, microvasculature network structure [6].

Micropatterning has also been applied to control 3D cellular alignment. In one study, Aubin et al. used photomasking to generate microengineered GelMA hydrogels and studied the effect of the feature sizes and aspect ratio of the engineered channels on the cell alignment and elongation behaviors in 3D gels [28]. GelMA hydrogels were photopatterned into rectangular microconstructs that were $150 \mu \mathrm{m}$ in height and down to $50 \mu \mathrm{m}$ in width. Compared with cells encapsulated in unpatterned GelMA hydrogels as the control, 3T3 cells showed enhanced alignment along the long axis of the rectangular microgels, and this effect was more significant in microconstructs with smaller widths. Several other cell lines such as 
HUVECs, rodent myoblasts (C2C12), and rodent cardiac side population cells (CSP) showed similar alignment and elongation behaviors in these patterned GelMA hydrogels [28]. Moreover, the cell-laden rectangular GelMA microconstructs were found to expand their widths, after initial swelling, due to cell proliferation at the external borders of the constructs. The authors showed that the gap between adjacent cell-laden constructs could progressively be filled with proliferating aligned cells. As a result, by properly designing the distance between neighboring microconstructs, self-assembled microtissues were achieved over the entire patterned surfaces, suggesting the possibility to fabricate highly aligned tissue constructs by using this simple strategy [28].

Fan et al. demonstrated a two-step photopatterning process to fabricate spatially defined 3D microgels based on GelMA to capture single neuron at the designed site of the microconstructs [40]. In the first step, GelMA hydrogels with gapped-loop shapes were prepared using a photomask and transferred onto a 3-(trimethoxysilyl)propyl methacrylate (TMSPMA)-coated glass slide. The micropatterned hydrogels were immersed into cell-laden GelMA solutions and underwent a second step photopolymerization to complete the entire loop structure, where individual cells could be captured at the gap position with moderate efficiency. It was found that a single neuron was able to spread after culturing within the engineered GelMA hydrogels, providing a practical tool to study axonal development [40].

Although photopatterning via photomask has been explored to fabricate 2D micropatterned hydrogels, this technique has limited capability to create microgels with complicated 3D architectures. Micro-mirror device projection printing systems have been 
developed to produce hydrogels with 3D microstructures via a layer-by-layer photopolymerization approach. Gauvin et al. reported the use of a custom designed projection stereolithography system to fabricate GelMA hydrogels with tunable microarchitectures (Figure 2B) [41]. They successfully obtained GelMA scaffolds with tunable mechanical properties (ranging from $<1-800 \mathrm{kPa}$ ) that depended on the designed 3D microstructures. The interconnected pore structures of the GelMA scaffolds were found to support adhesion and proliferation of HUVECs, where the cells reached confluency within a week [41]. When loading the proper computer-aided-design (CAD) files, this dynamic projection lithography technique allowed easy and rapid fabrication of GelMA hydrogels with different geometries to create 3D cell microenvironments similar to the native tissues [42]. Grogan et al. later tested the feasibility of using microfabricated GelMA hydrogels for meniscus tissue engineering applications [43]. They showed that the micropatterned GelMA constructs promoted the formation of meniscus-like tissue in a human ex vivo model by guiding the cellular alignment within biomimicking collagen bundles.

Two-photon polymerization (2PP) based on the phenomenon of two-photon absorption provides an alternative method to directly "write" micropatterns within hydrogels by using a femtosecond laser source. The 2PP technique allows localized excitation of the photoinitiator molecules and as a result, localized polymerization reaction that is controlled by the resolution of light exposure. Moreover, since the laser can be focused on any point of a transparent photosensitive material, there is no need for use of photomask or supporting materials, and the fabrication is not limited by the layer-by-layer approach. Complex 
architectures could be facilely obtained with proper CAD designs.

Ovsianikov et al. used 2PP laser fabrication to form complex GelMA microconstructs [44-46]. Potential cell damage related to the 2PP fabrication was studied by encapsulating osteosarcoma MG63 cells within the fabricated constructs. Cytotoxicity was found to correlate with the radical species generated during the initiation of photopolymerization. Major limitation of 2PP technique in fabricating cell-laden hydrogels is the relatively slow processing compared to other photopatterning methods. Due to the localized nature of the polymerization reaction, it could take hours to create a single structure, limiting 2PP large scale practical applications.

As a photopolymerizable biopolymer derivative, GelMA can be easily incorporated practically into all known photopatterning procedures. Various photopatterning technologies, such as the stereolithography, 2PP laser fabrication, and others to be developed, will continue to expand the applications of GelMA hydrogels in tissue engineering.

\subsection{Micromolded GelMA hydrogels}

Micromolding is a simple yet reproducible approach towards cost effective fabrication of patterned hydrogels with both planar and non-planar surfaces. Shape and feature sizes of the resulting micropatterns on the hydrogels are determined by the molds, which can reach a range of below 1 micrometer. Generally, the molds can be made from different materials, such as metal and plastics. Polydimethylsiloxane (PDMS) elastomers are also widely used to fabricate the molding replica due to their tunable mechanical strength, optical transparency, and biocompatibility. Surface properties of PDMS molds can be further modified to change 
the wettability of different liquids and promote easy detachment of the fabricated hydrogels. When the prepolymer solutions are placed in contact with the micropatterned mold, different crosslinking mechanisms can be used to form patterns within the gel. To fabricate patterned GelMA constructs, photocrosslinking is still the most convenient crosslinking strategy to transform the prepolymer solutions into hydrogels, and has been widely used in combination with micromolding techniques in many reports.

Qi et al. combined micromolding and photopatterning techniques to spatially organize 3D hybrid hydrogel arrays based on GelMA and PEG derivatives. Embryoid bodies (EBs) were encapsulated within the engineered constructs to study their interactions with different hydrogel compositions [47]. To do this, mouse EBs were first seeded into the microwells on a PDMS substrate, followed by the sequential loadings of the two prepolymer solutions and UV crosslinking through a photomask. The photomask was specifically designed so that the EB were embedded at the interfaces of the two different hydrogels and therefore were exposed to an asymmetric microenvironment. Due to the biodegradability of GelMA hydrogels, the EB were found to preferentially sprout into the GelMA hydrogel part and thus led to induced polarity in the embryonic development, suggesting possible approaches to regulate the behaviors of the stem cell niche for tissue regeneration applications.

In another study, Hosseini et al. fabricated a microgrooved GelMA substrate using PDMS molds and UV crosslinking techniques [10]. Murine C2C12 myoblasts were seeded within the microchannels of the grooved GelMA hydrogels and cultured in these pseudo-3D microenvironments. Both cell and myotube alignment were observed due to the geometrical 
confinement of the microchannels with no alignment on unpatterned GelMA hydrogels that were used as the control. Electrical stimulation was found to further promote the myotube alignment, facilitating the construction of a free standing contractile muscle tissue [10]. The effect of electrical stimulation could be enhanced by incorporating the microgrooved GelMA hydrogels with an interdigitated array of platinum $(\mathrm{Pt})$ electrodes [48]. It was found that electrical stimulation using the designed electrode assay resulted in a higher degree of $\mathrm{C} 2 \mathrm{C} 12$ myotube alignment $(\sim 80 \%)$ than using common Pt wire electrodes $(\sim 65 \%)$. In the meantime, larger myotube length, higher coverage area, and higher degree of protein biomarker expressions were also identified in the $\mathrm{C} 2 \mathrm{C} 12$ cells stimulated by this interdigitated array of Pt electrodes, suggesting the promises of GelMA hydrogels to engineer aligned muscle tissues [48]. A non-invasive glucose biosensor was later developed by Obregon et al. based on the cultured muscle tissue using a thin film of nanoporous gold [49]. By measuring the glucose uptake of the engineered skeletal muscle tissue with and without electrical stimulations, it was revealed that electrically stimulated muscle tissues consumed at least $10 \%$ more glucose when compared to normal tissues without electrical stimulation [49].

Microgrooved GelMA hydrogels with more complex surface patterns such as wavy or helical patterns have been fabricated using a fiber-assisted molding technique [50]. Nylon threads were aligned into plane assays or cylindrical helix by wrapping them onto a planar or tubular object, respectively. PDMS prepolymer solution was then coated and cured on the fiber patterns to generate the replicating molds for these patterns. The PDMS molds with unique surface features were used in the mold-patterning of GelMA hydrogels to transfer 
complex curved patterns to the surface of the resulting hydrogels ( $\mathrm{ca} .30 \%$ higher number of aligned myotubes at day 8). C2C12 myoblasts cultured on these GelMA hydrogels patterned with sinusoidal grooves showed improved cell alignment when compared with myoblasts cultured on conventional channel-ridge patterned hydrogels. This indicated promising potential applications of grooved GelMA hydrogels with complex patterns in achieving in vitro cellular alignment [1]. Similarly, Annabi et al. applied micromolded GelMA hydrogels with channel-ridge patterns to culture cardiomyocytes in vitro [51]. Enhanced cell alignment and contractile activity of the cardiomyocytes were demonstrated on micropatterned GelMA hydrogels, compared to unpatterned ones. After 72 hours of culture, $42 \%$ of the cells were aligned in the micropatterned GelMA versus $23 \%$ in unpatterned GelMA [51].

Micromolding has been combined with other fabrication techniques to engineer vessel-like structures. Sadr et al. produced GelMA blocks with a cylindrical lumen by casting GelMA hydrogel around a gold rod [52]. The rod surface was decorated with a layer of HUVEC and NIH 3 T3 cells. The cell layer was released from the rod and printed onto the GelMA surface by applying voltage $(1 \mathrm{~V})$ to the rod ends. Removal of the rod from the block of GelMA left behind a vessel-like structure. Further cell proliferation at the vessel walls was promoted by continuous perfusion of culture medium.

One of the important features of the micromolding technology is the ability to create microfabricated hydrogels with complex 3D structures with high resolution. The combined use of templates and photocrosslinking provides a powerful and straightforward strategy to engineer high resolution complex GelMA microstructures. 


\subsection{Self-assembled GelMA hydrogels}

Self-assembly has been exploited to create microstructural features in GelMA based scaffolds. For example, self-assembly of cell-laden GelMA hydrogels of different geometries with micro-scale range (i.e., cubes of around $1000 \mu \mathrm{m}^{3}$ in volume) was demonstrated by placing the cubes of GelMA, onto the surface of a higher density hydrophobic liquid (i.e., $\mathrm{CCl}_{4}$ or perfluorodecalin). Formation of complex aggregates of the microgels was observed, due to surface tension that drove minimization of the free energy of the system (Figure 2C) [53]. These aggregates can be consolidated by a second crosslinking step using UV light irradiation. This simple technique is powerful in the context of building complex and relevant tissue engineering architectures. The microgel blocks can encapsulate different cell types, which could be aggregated to design multi-cellular structures.

Precise control of the assembly process can be achieved by functionalizing the surface of the cell-laden hydrogel blocks. For example, Qi et al. fabricated cube-shaped hydrogels functionalized with single-stranded DNA segments [54]. The authors showed that cubes loaded with long complementary DNA strains could preferentially bind to each other. Consequently, local aggregations and thus, the microarchitectures, could be directed by using cell-laden hydrogel blocks with complementary DNA strands [54].

An alternative strategy to take advantage of the self-assembly phenomenon in tissue engineering applications is the use of magnetic forces or magnetic alignment. In one embodiment of this strategy, Xu et al. fabricated magnetic GelMA and PEG micro-hydrogels and aligned them using a chamber with an array of magnetic rods [55]. The authors showed 
that complex 3D structures, composed of these magnetic microgels, could be assembled using simple strategies. For example, sequential immersion of a magnetic rod in three different magnetic microgel suspensions resulted in the fabrication of a structure composed of three sequential layers of cell-laden hydrogels. Tasoglu et al. have recently shown that cell-laden hydrogel structures (i.e., GelMA hydrogels) can be assembled, in a contactless manner, using a levitation magnetic-based strategy [56]. The authors showed that hydrogel building blocks with complementary geometry can be easily self-assembled using magnetic levitation when floating in a paramagnetic solution (prepared by addition of gadolinium ions).

As a spontaneous process driven by thermodynamics, self-assembly is a useful "bottom-up" approach to obtain complex 3D architectures from the controlled aggregation of sub-units or building blocks. However, the study of microgel self-assembly towards ordered structures is in its early stage, and precise control over the self-assembly process to mimic the delicate structures of native tissues is still an important challenge. Introduction of complementary DNA strands has been a promising solid step towards this goal. Designing and understanding new self-assembling microgel systems will provide useful insights and tools to further develop tissue engineering platforms.

\subsection{GelMA hydrogels in microfluidics}

Microfluidics refers to a broad, multidiscipline research field with the focus on designed systems involving the flow of small volume of fluids. In tissue engineering, microfluidic devices have been applied to fabricate various shaped microgels (e.g. microfibers or microparticles), organ-on-a-chip platforms, and hydrogels containing gradients. Here we 
summarize some studies that combine microfluidic devices with GelMA hydrogels for different bioengineering applications.

Hancock et al. reported a simple technique to generate concentration gradients in photocrosslinkable hydrogels (including GelMA hydrogels) over centimeter length scale [57]. A stripe of prepolymer solution was applied to a mold with hydrophobic boundary, followed by the addition of a drop of solution containing the targeting material at one end of the strip. Capillary flow drove the diffusion of the molecules, nanoparticles, or even cells within the stripe, which was controlled by a few parameters, such as geometric dimension of the stripe, viscosity of the fluid, and surface tension. Cell spreading gradient was achieved by controlling the concentration gradient of GelMA along the photocrosslinked hydrogel stripe [57]. Later, Piraino et al. further developed this technique to generate GelMA hydrogels with multiple gradient layers by sequentially repeating the procedure and a layer-by-layer process. Notably, the gradients could be in parallel or inverse directions along the hydrogel stripe [58].

Chen et al. developed a microfluidic platform with GelMA hydrogels to investigate the cardiovascular cell-cell interactions within the channels under 3D physiological conditions [11]. Bilayered microchannel devices were fabricated to incorporate valvular endothelial cells (VECs) in the top layer and VICs embedded in GelMA hydrogels in the bottom layer, which were separated by a porous membrane. The interactions between VECs and VICs regulated by shear forces were studied using this platform. It was showed that VECs suppressed VIC myofibroblast differentiation, and this effect could be further enhanced by applying shear stress only to the VECs on the top layer. These results were similar to the behaviors observed 
in macroscale systems, implying that this microfluidic device could be used for cost-efficient and high-throughput screening studies [11].

Hiesh et al. reported the observation of 3D alignment of NIH $3 \mathrm{~T} 3$ fibroblasts by applying gradient compressive forces to cell-laden GelMA hydrogels with a height gradient within a designed microfluidic device [59]. A PDMS membrane covered the concentric circle-patterned cell-laden hydrogels, and generated a gradient strain that was maximized in the center of the circle. Encapsulated NIH $3 \mathrm{~T} 3$ cells demonstrated a trend of alignment that positively correlated to the applied strain on the hydrogels. This system provided a facile platform to investigate cellular behaviors (viability, proliferation, and alignment) under a gradient of external compressive strain [59].

Annabi et al. developed a technique to coat microfluidic devices with photocrosslinkable hydrogels (such as GelMA) and used the coated devices to generate a heart-on-a-chip platform, which can be used for drug screening applications. GelMA prepolymer solution was injected through PDMS cylindrical microchannels at a constant rate between 0 and 20 $\mu \mathrm{L} / \mathrm{min}$, while being illuminated with UV light to achieve crosslinking (Figure 2D). The thickness of the GelMA coating could be adjusted by varying the feeding flow rate, the intensity of the UV light, and/or the concentration of the GelMA prepolymer solution. Moreover, GelMA precursor and cells can be mixed to fabricate microchannels coated with cell-laden GelMA hydrogels [60].

Incorporating GelMA hydrogels within microfluidic devices offer the possibility to study cell-biomaterials interactions under defined microenvironments. The continuous flow 
within the devices can provide the cells with similar conditions to those found in their native tissues (e.g., oxygen and nutrient supply, certain shear stress level, controlled exposure to certain stimuli), while the GelMA hydrogels serve as excellent ECM-mimicking materials for the encapsulated cells. It is easy to anticipate that GelMA hydrogels will soon find additional niches in microfluidic applications such as those related to biosensing and drug screening.

\subsection{Bioprinted GelMA hydrogels}

Bioprinting derives from the concept of additive manufacture, where a 3D architecture can be fabricated by a predesigned and sequential deposition of layers of a "bioink" (such as in an ordinary $3 \mathrm{D}$ printing process). Bioprinting aims to accurately dispense cells, hydrogels, or cell-laden materials, to achieve spatially controlled 3D constructs and to mimic the structures of targeting tissues. Cell stability and viability should be retained during the printing process, by carefully designing biocompatible materials and crosslinking methods, and optimizing bioprinting parameters. The assembled cell-laden constructs provide tissue-like environments for the cells to proliferate and finally mature into a tissue.

Bioprinting technologies have been applied to generate GelMA-based 3D constructs with controlled architectures for various tissue engineering applications. For example, Schuurman et al. reported that GelMA solutions mixed with chondrocyte cells were printed into multiple layered network structures, where high viability of cells $(83 \pm 13 \%$ and $73 \pm 2 \%$ at day 1 and 3, respectively) were observed within the GelMA hydrogels. The authors also studied the effect of other additives, such as hyaluronic acid (HA), on the viscosity of the GelMA solution to facilitate the printing procedure [15]. Hoch et al. studied the use of 
chemically tailored GelMA in the piezoelectric inkjet printing technique. They further modified GelMA with a second acetylation reaction to tune the solution viscosity and physical property, and to improve its processibility. Cell-laden spot arrays were formed where the encapsulated porcine chondrocytes exhibited high viability within the hydrogel [61]. Other studies also have explored the use of GelMA hydrogels in the bioprinting technique to manufacture cell-laden constructs with high cell viability (between 77 and 97\%) for tissue engineering applications $[18,62]$.

Bertassoni et al. reported that GelMA solutions with a range of concentrations ( 7 to $15 \%$ ) can be mixed with cells and extruded through a modified bioprinter (Organovo ${ }^{\mathrm{TM}}$ ) mounted into a robotic $\mathrm{X}-\mathrm{Z}$ system to fabricate cell-laden cylinders with varying cell densities (Figure 2E) [12]. The key modification consists of the addition of a UV light guide (Omnicure S2000) that could trigger the photocrosslinking of the cell-polymer suspensions inside a glass capillary tube. A metallic micro-piston allowed the automatized formation of cell-laden cylinders of GelMA. The coordinated motion of the motorized X-Y-Z stage enabled precise printing and arrangement of the cell-laden GelMA cylinders into fibers or piles [12].

Bioprinting is an important addition to the toolbox of scaffold engineering, both on its own and in combination with other techniques. Bioprinting and 3D molding can be used to design cell-laden hydrogels with embedded cylindrical microchannels that resemble vasculature, a key element for close mimicry of complex and functional tissue architectures. Cylindrical-shaped segments of agarose, a natural polysaccharide, were also bioprinted and 
used as sacrificial template to form vascularized cell-laden GelMA hydrogels (Figure 2E) [63]. The sacrificial agarose segments could be easily removed from the GelMA matrix by simply pulling of the agarose cords, resulting in a bioprinted GelMA scaffold with internal hollow "vessels" [63].

Recently, Kolesky et al. reported similar bioprinting approaches for the creation of vasculature within GelMA hydrogels [64]. They designed several different inks compatible with the printing process to generate complex constructs of multiple types of cells as well as hollow channels. A triblock copolymer of polyethylene glycol (PEG) and polyproprylene oxide (Pluronic F127) was used to print the fugitive filaments that could be easily removed to create the hollow vascular network structure and allow HUVECs to perfuse in and form vessels. Vascularized, heterogeneous tissue constructs with spatially positioned cells were created within a GelMA matrix by using this strategy [64].

Bioprinting allows feasible and precise processing of biomaterials and cells to generate spatially-controlled distributions in 3D. Provided that the goals of preserving cell viability and function are achieved, bioprinting can provide a solution to the fabrication of complex cell-laden constructs that mimic the function of certain tissues and lead to the direct manufacturing of mature artificial tissue in vitro that are suitable for transplantation. The excellent biocompatibility, degradability, and processibility of GelMA hydrogels will play an important role, as a printable biomaterial, in 3D bioprinting technologies.

\subsection{GelMA hydrogel fibers and fabrics}

Textile techniques, originally aimed at generating clothing and decorative fabrics, can 
produce complex constructs with tunable mechanical properties with precise control over patterns. Recently, these techniques have been extensively used for engineering tissue engineering scaffolds with biomimetic mechanical properties [65-68]. These efforts also has been fueled by the similarity of the morphology some tissues such as tendons, ligaments, and muscles to the generated fabrics. However, the full potential of textile technologies in tissue engineering can be achieved by assembling cell-laden fibers. Hydrogels in general and GelMA is particular are excellent cell carriers, but there are only a few experiments on the fabrication of GelMA fibers and fabrics. Fabrication of GelMA fibers is not trivial as the crosslinking process is not very rapid and the need for integration of a UV illumination system with the fiber spinning system makes the fabrication process challenging. In addition, the mechanical properties of GelMA are also not favorable for use in textile processes.

In a recent study, Shi et al. generated GelMA fibers by injecting the pre-polymer solution into a cold solution to solidify the fibers first and then chemically crosslinked them using UV illumination [69]. However, the rapid dispersion of GelMA into the cold solution significantly affected the controllability of the process. In another approach Tamayol et al. used sacrificial alginate templates for engineering GelMA fibers. In this approach GelMA pre-polymer was mixed with alginate and then wetspun into $\mathrm{CaCl}_{2}$ solution to crosslink alginate and physically entrap GelMA prepolymer. Fibers were then exposed to UV light to crosslink GelMA and alginate was removed using a calcium chelator. The presence of alginate enhanced the mechanical properties of the generated hybrid hydrogels and for the first time they were able to generate woven and braiding fabricated from GelMA with clinically relevant dimensions 
(Figure 2G).

\section{Hybrid hydrogels based on GelMA}

Hybrid hydrogels are constructed from blends of different components, which are selected to possess specific properties. Although GelMA is a cell-responsive material with wide-spectrum of tunable properties, tailored design of hybrid materials has been a useful strategy to improve some characteristics of GelMA for certain applications. Table 1 summarizes some examples of hybrid hydrogels based on GelMA and their targeting properties [1]. In this section, we review hybrid hydrogels based on GelMA and other constituents including carbon nanotubes (CNT), graphene oxide (GO), inorganic nanoparticles, other biopolymers, and synthetic polymers.

\subsection{Carbon nanotubes (CNTs), graphene oxide (GO), and graphene incorporated}

\section{GelMA}

Some tissue engineering applications require matrices with mechanical strengths higher than those normally obtained from GelMA hydrogels (up to $\sim 30 \mathrm{kPa}$ in compressive modulus). The use of GelMA prepolymer solutions with higher polymer concentrations (> $15 \%$ ) can lead to enhanced mechanical properties of GelMA hydrogels, but at the expense of affecting other relevant characteristics such as degradability, porosity, and 3D cellular spreading and growth. As a result, developing hybrid hydrogels based on GelMA has been used as an alternative to enhance the mechanical strengths of GelMA-based hydrogels, which still serve as suitable scaffolds for cell growth (Figure 3A).[70, 71]

Shin et al. added CNTs into GelMA hydrogels to enhance their stiffness without 
decreasing their porosity or inhibiting 3D cellular growth. 3T3 fibroblasts exhibited adequate growth in GelMA hydrogels containing CNTs in a range of concentrations from $0.1-0.5$ $\mathrm{mg} / \mathrm{mL}$ [70]. GelMA-CNT hydrogels showed a compressive modulus of $31 \pm 2.4 \mathrm{kPa}$ for 5 w/v\% GelMA at the concentration of $0.5 \mathrm{mg} / \mathrm{mL} \mathrm{CNT}$, which was far superior to that observed for pristine $5 \mathrm{w} / \mathrm{v} \%$ GelMA hydrogels $(10 \pm 0.5 \mathrm{kPa})$ [71]. 3D cell culture experiments showed statistically similar viabilities (between 85-100\%) with those observed in the controls (no CNTs added) after 24 and 48 hours of seeding.

Incorporation of CNT could also fine-tune the electrical conductivity of the resulting hydrogels [71], which is an important characteristic in myocardial or neuronal tissue engineering applications. For instance, myocardial cells cultured on $50-\mu \mathrm{m}$-thick GelMA-CNT hydrogels demonstrated better alignment behaviors, 3-fold higher synchronous beating rates, and an $85 \%$ lower excitation threshold, when compared to cells cultured on the surface of pristine GelMA hydrogels [71].

Electrical signals can be used to align CNTs within the GelMA matrix and develop well-defined hybrid hydrogel architectures to direct cell growth. For example, Ramón-Azcón et al. developed nanostructured GelMA-CNT hydrogels within which the CNTs were aligned using dielectrophoresis (DEP) forces [72]. At a given voltage, the current intensity conducted through the GelMA hydrogels could be increased up to two orders of magnitude by the addition of $0.1 \mathrm{mg} / \mathrm{mL} \mathrm{CNTs,} \mathrm{while} \mathrm{alignment} \mathrm{of} \mathrm{the} \mathrm{CNTs} \mathrm{further} \mathrm{increased} \mathrm{the} \mathrm{conductivity}$ by three orders of magnitude. GelMA-CNT hybrid hydrogels with improved conductivity could be used to control cell growth and protein expressions. Muscle cells grown on aligned 
GelMA-CNT hydrogels yielded a higher number of functional myofibers when compared with those cultured on hydrogels with randomly distributed CNTs, as confirmed by the enhanced expression of myogenic genes (at least two-fold higher expression of myogenin, $\alpha$-actinin, and MRF4) [73]. This effect could be further amplified when electrical stimulation was applied along the direction of CNT alignment, due to the oriented conductivity of the aligned GelMA-CNT hydrogels [73].

Graphene oxide (GO) has also been used as an effective additive to enhance the mechanical properties of GelMA hydrogels. Shin et al. demonstrated that GelMA-GO hybrid hydrogels could facilitate spreading and alignment of cells, and improve viability and proliferation in $3 \mathrm{D}$ microenvironments, probably due to the interactions between cells and the GO nanostructures within the composite [71]. The GO reinforcement technique was applied in combination with a multiple layer stacking approach towards the facile construction of complex artificial tissues with mechanical stability and cytocompatibility. The GelMA-GO hybrid hydrogels also provided the capability to fine-tune the mechanical and electrical properties without compromising the processibility for microfabrication. Cell-laden constructs could also be fabricated from GelMA-GO gels with higher GO concentrations (up to $2.0 \mathrm{mg} / \mathrm{mL}$ ) than the GelMA-CNT gels without affecting the crosslinking density [71].

Reacting GO with 3-(trimethoxysilyl)propyl methacrylate afforded methacrylated graphene oxide (MeGO) as an alternative candidate to improve the stability of GO particles within the hydrogel matrix [74]. Cha et al. reported that hybrid GelMA-MeGO hydrogels exhibited superior toughness and a higher resistance to fracture when compared to 
GelMA-GO and pristine GelMA hydrogels (Figure 3B) [74]. GelMA-MeGO hydrogels $(3 \mathrm{mg} / \mathrm{mL}$ of $\mathrm{MeGO})$ exhibited an 11-fold increase in ultimate stress when compared to GelMA-GO analogues. The proliferation rate of fibroblasts in GelMA-MeGO hydrogels was comparable to that observed in GelMA-GO analogues and significantly superior (at least $60 \%$ higher) to that found in pure GelMA hydrogels [74].

GelMA impregnated with polyethylenimine (PEI) functionalized GO nanosheets (fGO) has been also used to achieve site-specific gene delivery to infarcted cardiac tissues in a rat model [75]. In particular, Paul et al. engineered an injectable formulation of the hybrid GelMA-fGO hydrogel carrying a pro-angiogenic human vascular endothelial growth factor plasmid DNA (pDNAVEGF) bound to fGO to facilitate local myocardial neovascularization at the injected sites. Transfected cells showed significant mitotic activities. In addition, this gene therapy induced a significant increase in myocardial capillary density at the injected infarcted region and a decrease in the scar extension when compared to animals treated with GelMA-fGO hydrogels without DNA, GelMA hydrogels with VEGF-DNA but without functionalized GO nano sheets, or pristine GelMA hydrogels [75].

Ahadian et al. have proposed a facile and green method for the production of graphene dispersions and their incorporation into a GelMA hydrogel to enhance its stiffness (the Young's modulus increased from $33.5 \pm 6.7 \mathrm{KPa}$ in pristine GelMA to $45.8 \pm 9.9 \mathrm{KPa}$ in GelMA graphene hydrogels containing $0.1 \mathrm{mg} / \mathrm{mL} \mathrm{GO}$ ) and conductivity (an increase of up to two orders of magnitude with respect to pristine GelMA) [76]. The addition of carbon nanomaterials such CNT and GO into GelMA hydrogels not only improves their mechanical 
properties, but also enhances the electrical conductivity of the resulting hybrid hydrogels. This provides a simple strategy to produce scaffolds for in vitro cell culture studies in which the application of an external electrical stimuli might be required (i.e. in the engineering of myocardial tissues and skeletal muscle tissues).

\subsection{GelMA hydrogel containing inorganic nanoparticles}

The addition of gold [77, 78] and other inorganic particles [79, 80] has been used to improve the mechanical strengths [81], conductivity [77], response to thermal or magnetic stimuli, and biological functionality of GelMA hydrogels [78]. For example, Heo et al. used gold nanoparticles (GNP) as additives to GelMA hydrogels for bone tissue engineering applications (Figure 3C) [78]. They demonstrated that GelMA-GNP hydrogels promoted proliferation and differentiation of human adipose-derived stem cells (ADSCs) into osteoblast cells, as indicated by increased alkaline phosphatase (ALP) activity levels (up to 85\% increase at day 14) and significantly increased expression of three genes related to bone differentiation, namely, bone sialoprotein (BSP), osteocalcin $(\mathrm{OCN})$, and runt-related transcription factor 2 (RUNX2) [78].

GelMA-hydroxyapatite hybrids was also proposed as coatings for titanium (Ti) surfaces to improve the integration between titanium and bone implants [82]. In this application, the titanium surfaces were first hydroxylated by alkali treatment and then covalently coated with a GelMA film. Immersion of the GelMA-coated Ti constructs into a solution that mimics concentrated human plasma $(2 \mathrm{X})$ for three days induced mineralization and led to the formation of a hydroxyapatite-GelMA layer [82]. 
Recently, Xavier et al. reported the formation of GelMA-based hybrid hydrogels containing 2D nanosilicates [83]. The inorganic compositions, high anisotropy, and large surface area of the nanosilicates [84] enhanced the interactions between GelMA hydrogel and encapsulated cells. A four-fold increase in compressive modulus, as well as increased pore size (from 0.2-0.9 $\mu \mathrm{m}$ to $0.3-1.5 \mu \mathrm{m}$ ), was observed for the hybrid hydrogels when compared to pristine GelMA hydrogels. Moreover, in vitro culture of preosteoblast NIH MC3T3 cells revealed that the hybrid hydrogels could promote osteogenesis even in the absence of osteoinductive growth factors in the media, as supported by a three-fold increase in ALP activity and a four-fold increase in mineralized matrix formation. These results suggested a promising growth-factor-free approach towards bone tissue engineering [83].

\subsection{Hybrid hydrogels based on GelMA and other biopolymers}

The fabrication and use of GelMA hybrids with other biopolymers has been explored for diverse purposes, including enhancing mechanical properties $[85,86]$, tuning characteristics such as porosity, swelling ratio and degradability [86], or imparting a particular property such as electrical conductivity $[71,87]$.

Preparation of hybrid hydrogels based on hyaluronic acid methacrylate (HAMA) and GelMA have received particular attention (Figure 3D). Since HA and collagen are the major ECM components in most tissues (such as cardiovascular, cartilage, neural tissues), the GelMA-HAMA hybrid hydrogels would naturally be a promising candidate to closely mimic the ECM of native tissues. A recent characterization of the physical properties of GelMA-HAMA hybrids, including swelling ratio, degradation, mechanical properties, and in 
vitro cellular responses, revealed significantly improved mechanical properties when compared to the single component analogues. For instance, the compressive modulus of 5\% GelMA hydrogels increased from 4 to $36 \mathrm{KPa}$ when $2 \%$ HAMA was added. Similarly, the compressive modulus of $10 \%$ GelMA hydrogels was increased from 32 to $72 \mathrm{KPa}$ by adding $2 \%$ HAMA [88].

Levett et al. also explored the hybrid hydrogels based on GelMA and HAMA, as well as chondroitin sulfate methacrylate (CSMA)-GelMA hybrids, for cartilage tissue engineering [85]. The authors encapsulated human chondrocytes in GelMA-HAMA and GelMA-CSMA hydrogels and observed that both materials were able to enhance chondrogenesis as evaluated by gene expression assays and immunofluorescence [85]. The addition of HAMA to GelMA constructs resulted in rounder cell morphologies and increased the quantity and distribution of the newly synthesized ECM throughout the construct. This exacerbated process of chondrogenesis increased the compressive modulus of the GelMA-HAMA and GelMA-CSMA constructs by $114 \mathrm{kPa}$ after 8 weeks of culture, while the control GelMA constructs increased by $26 \mathrm{kPa}$ during the same time period. Recently, a GelMA-HAMA composite hydrogel has been suggested as a system for studying the processes of differentiation of valvular tissue interstitial cells (VIC); the use of this research platform can provide relevant information that would aid in understanding the pathobiology of valvular disease [89].

The construction of interpenetrating polymer network (IPN) composed of GelMA and silk fibroin (SF) has also been explored. Silk on its own is an attractive material for tissue 
engineering applications because of its easy processing and high mechanical strength. Xiao et al. synthesized and characterized GelMA-SF IPN hydrogels by a simple two-step process consisting of a photocrosslinking reaction of the mixture of GelMA and silk fibroin under UV light, and a subsequent treatment with a $70 \%$ methanol solution to promote crystallization of silk fibroin within the hydrogels [31]. The resulting hybrids were characterized in terms of their mechanical properties, swelling ratio, biodegradability, and potential use as 3D scaffolds.

The use of GelMA as a component of a dual-interpenetrated polymer network (DN) has also been reported. For example, Shin et al. developed high strength DN hydrogels (with a compressive modulus up to $\sim 90 \mathrm{kPa}$ and compressive failure stress up to $\sim 6.9 \mathrm{MPa}$ ) capable of encapsulating cells (NIH-3T3 fibroblasts) by a two-step photocrosslinking process using two modified biomacromolecules: gellan gum methacrylate (GGMA) and GelMA [90]. A viability assessment of NIH-3T3 cells cultured on the engineered materials ( $82 \%$ and $71 \%$ at day 0 and 3, respectively) demonstrated that the entire process of DN network formation was cell-friendly and could potentially be used to produce scaffolds that closely mimic the mechanical properties of tissues such as cartilage or tendons [90].

The addition of a second crosslinkable biopolymer component to GelMA would expand its potential applications by improving the mechanical properties of pristine GelMA hydrogels as well as by adding new functionalities. For example, Bae et al. observed that cells encapsulated in pullulan methacrylate (PulMA) hydrogels did not elongate but organized into clusters with tunable cluster sizes controlled by hydrogel compositions (i.e., 
by different mixing ratios) [91]. These data suggest that GelMA-PulMA hydrogels are potentially useful for fabricating cell-responsive microenvironments (micro-tissues) for applications that favor controlled cell clustering and/or localized proliferation. Other GelMA-containing dual-biopolymer network composites reported to date include GelMA with dextran glycidyl methacrylate (DexMA). GelMA-DexMAs composites with a high degree of substitution show a lower swelling ratio ( $\sim 25 \%$ lower) and a higher compressive modulus ( 4.7 fold higher) than their GelMA counterparts [86]. Furthermore, Liu et al. demonstrated that hydrogels made from mixtures of DexMA (functionalized with lysine) and GelMA exhibit promising properties for use in vascular tissue engineering [19]. These authors tuned the shear storage modulus in the range of $c a$.900-6000 Pa by: (1) controlling the degree of MA and lysine substitution in the biopolymers, and (2) changing the DexMA/GelMA mass ratio in the hybrid hydrogel. Artery smooth muscle cells encapsulated in hybrid hydrogels with a shear storage modulus in the range of 898-3124 Pa were able to proliferate, spread, and form vascular networks [19].

In yet another example of the versatility and diversity of GelMA hybrids, Li et al. presented a technique to micro-pattern GelMA hydrogels using a collagen mimetic peptide (GMP) that contains photo-labile nitrobenzyl groups [92]. GMP attached to the gelatin chains in GelMA by non-covalent affinity, and could be further photocrosslinked within the pre-existing GelMA matrix, thereby providing a flexible tool for the creation of micro-patterns in GelMA hydrogels.

In a recent contribution, Visser et al. fabricated GelMA hydrogels loaded with embedded 
equine cartilage-derived matrix (CDM) particles [93]. They demonstrated that this addition of CDM particles to GelMA significantly stimulated the formation of a cartilage template by MSCs in a GelMA hydrogel-based MSC culture system.

The fact that the native ECM is by itself a highly heterogeneous environment composed of numerous components further justifies the idea of developing hybrid hydrogels including more than one biopolymer. The combination of GelMA with other natural polymers offers additional dimensions for tuning the properties of hybrid hydrogels towards ECM-mimicking scaffolds. Future studies might focus on optimizing the compositions of GelMA-based hybrid hydrogel systems to meet the requirements of specific tissue engineering applications.

\subsection{Hybrid hydrogels based on GelMA and synthetic polymers}

Several authors have reported the synthesis and characterization of hybrid hydrogels based on synthetic polymers and GelMA [6, 7, 94-96]. For example, Hutson et al. reported the preparation of hybrid hydrogels composed of GelMA (up to $15 \%$ w/v) and PEG (up to $10 \%$ w/v) [7]. The combination of GelMA and photocrosslinkable PEG derivatives provided hybrid hydrogels with tunable physical and biological properties. Adding GelMA (5-15\% $\mathrm{w} / \mathrm{v})$ to PEG (5 and $10 \% \mathrm{w} / \mathrm{v})$ proportionally increased fibroblast surface binding affinity and spreading, when compared to pure PEG hydrogels [7]. For instance Hutson et al. observed that the area coverage of fibroblasts cultured on PEG hydrogel surfaces was less than $1 \%$ after six hours of culture. In contrast, fibroblasts growing on $10 \%$ GelMA-10\%PEG covered more than $10 \%$ of the area of culture within the same time period. Encapsulated fibroblasts in the GelMA-PEG hybrid hydrogels could form 3D cellular networks after culturing for 7 days, 
which was not observed in pure PEG hydrogels [7].

In another study, Qi et al. exploited the differences between GelMA and PEG to control the bipolar environments that dictated cell growth. They combined micromolding and photomasking techniques to spatially organize 3D hybrid hydrogel arrays based on GelMA and PEG derivatives (Figure 3F) [47]. EBs derived from mouse embryonic stem cells (ESCs) exhibited polarized developments with patterned vasculogenic differentiation. Importantly, the polarized differentiation of each EB in the engineered bipolar ECM was associated with potential cellular interactions between the different portions of the EBs exposed to different microenvironments [47].

Nanopatterned hybrid scaffolds, based on GelMA, HA, and PEG dimethacrylate, were studied by Nemeth et al. [97] with the aim of inducing chondrogenesis from dental pulp stem cells (DPSCs). Morphological observations, together with gene and protein expression analysis, showed that both nanopatterning and the addition of HA component drove DPSCs to undergo chondrogenic differentiation.

Similarly, Pedrón et al. used PEG-GelMA-HAMA hydrogels as a model matrix to mimic the glioma in order to study human glioblastoma multiforme (hGBM), the most common and aggressive form of brain cancer [98]. In another study, Serafim et al. characterized a family of hybrid hydrogels made from GelMA and polyacrylamide (PAA) by a simple "one-pot" synthesis using photopolymerization [17]. The authors presented a correlation between composition and hybrid PAA-GelMA properties relevant to tissue culture and drug delivery applications, such as swelling, mechanical properties, porosity of the resulting covalent 
PAA-GelMA network, and biodegradability [17].

Boere et al. reported a fairly elaborated process to prepare novel hybrid materials by grafting poly-methacrylated-poly(hydroxymethylglycolide-co- $\varepsilon$-caprolactone) -poly $(\varepsilon$ -caprolactone) (pMHMGCL-PCL) to the surface of GelMA hydrogels (Figure 3E) [99]. The covalent bonding between these two materials (modified PCL and GelMA) yielded an improved resistance to repeated axial and rotational forces at the interface. Human chondrocytes embedded within the constructs were able to form cartilage-specific matrix in vitro, as suggested by immunostaining results, after six weeks of culture. Remarkably, the implantation of these constructs in rats resulted in extensive deposition of collagen II, including deposition at the interface between the implant and the native tissues [99].

Specific high-end applications require materials with specific characteristics. In these cases, hybrid hydrogels based on the polymer and GelMA mixtures of greater complexity have been tested. For example, in the context of heart valve engineering, materials have to tolerate the dynamical stresses of the heart valve microenvironment. To obtain hybrid scaffolds with enhanced mechanical properties, electrospun poly(glycerol sebacate) (PGS)/poly( $\varepsilon$-caprolactone) (PCL) microfiber scaffolds were integrated within a hybrid hydrogel made from HAMA-GelMA [100]. Sheep mitral VICs were encapsulated in this hybrid hydrogel. The 3D distribution of the cells was more homogeneous than those found in pristine GelMA hydrogels or microfiber scaffolds without GelMA. Compared to electrospun fibers or hydrogel scaffolds alone, the hybrid system offered a suitable 3D matrix for heart valve tissue engineering. 
Current controlled polymerization techniques and the diverse nature of available synthetic polymers provide unlimited opportunities to achieve desired properties in hybrid hydrogels by combining GelMA and other synthetic polymers. More importantly, the mixing of synthetic polymers and GelMA can take place at the molecular level by directly mixing them as the prepolymer solution, or at the supramolecular level by incorporating engineered synthetic polymer assemblies into the GelMA hydrogel matrix, such as the microfibers embedded in GelMA hydrogel as reinforcement fillers [100]. These possibilities further expand the future applications of hybrid GelMA hydrogels in biomedical field.

\section{Biomedical applications of GelMA hydrogels}

\subsection{Tissue engineering}

In general, GelMA possesses many relevant characteristics to serve as tissue engineering scaffolds. GelMA-based hydrogels are biocompatible, biodegradable, non-cytotoxic, and non-immunogenic. GelMA is also a versatile biomaterial with tunable physicochemical properties, promising remarkable compatibility for a wide spectrum of applications. In addition, the photocrosslinkable feature of GelMA enables flexibility for microengineering by different microfabrication methods as explained previously [1]. It has been shown that different cells adhere to and grow on the surface of GelMA substrates, and can be encapsulated within the GelMA hydrogel matrixes with excellent viability [6, 29]. As a result, the versatility of GelMA hydrogels to address the specific tissue engineering demands (Figure 4) and to produce tissues for clinical, diagnostic, or pharmaceutical research purposes have been intensively explored. 
Aubin et al. grew different types of cells on micropatterned GelMA hydrogels to form different tissues: HUVECs for vascularization, rodent myoblasts $(\mathrm{C} 2 \mathrm{C} 12)$ for skeletal muscle, cardiac side population cells (CSPs) for myocardial tissue, and NIH 3 T3 fibroblasts as a generic model [28]. They demonstrated cells could proliferate, align, and elongate on GelMA by following the guidance of the micropatterned hydrogel architectures. This type of growth is especially desirable for vascular, muscle, and cardiac tissues since their functionality is greatly related to their ability to form aligned fibers responsive to mechanical or even electrical stimuli. Since then, micropatterned GelMA hydrogels have been widely used to tune the cellular microenvironment and control the cellular behaviors for tissue engineering studies $[6,8,28,51]$.

The development of vascular networks is an important aim in tissue engineering. Vascularization is the key requirement for the engineering of functional 3D tissues such as heart, liver, kidney, and bone, since it provides the surrounding engineered tissues with nutrients and oxygen, and facilitates the removal of metabolic byproducts [9, 63]. GelMA hydrogels have been successfully used in several studies for the development of vascular networks in 3D environments. For instance, Nikkhah et al. produced endothelial cord structures with a circular cross-section using micropatterned GelMA constructs (Figure 5A) [8]. They produced GelMA constructs with different heights $(50,100$, and $150 \mu \mathrm{M})$ and found that geometry has an important effect on the cellular behaviors to form organized and stable cord structures over different culture times. Constructs with $100 \mu \mathrm{M}$ in height provided the optimal microenvironment to produce endothelial cord structures [8]. 
Chen et al. [9] and Lin et al. [13] demonstrated the use of GelMA hydrogels containing endothelial colony-forming cells (ECFCs) and mesenchymal stem cells (MSCs) as scaffolds for vascular morphogenesis in vitro and in vivo. Both studies reported the formation of vascular networks with functional anastomoses integrated to the mouse vasculature. Lin et al. directly injected GelMA prepolymer solutions loaded with ECFCs and MSCs into mice, and polymerized the gel in situ by transdermal exposure to UV light [13]. After 7 days, they explanted the GelMA hydrogels and observed formation of stable and interconnected vasculature uniformly distributed within the scaffold (Figure 5B) [13]. Recently, our group has shown that bioprinted GelMA hydrogels provided a favorable microenvironment for HUVECs to form a confluent monolayer, and for MC3T3 cells to differentiate into osteogenic cells [12]. In these studies, fine control of the microarchitecture is a valuable tool for the production of vascularized 3D tissues.

Engineering of cardiac constructs using GelMA-based hydrogels has also been reported. Different strategies have been employed to tune relevant hydrogel properties for cardiovascular tissues such as mechanical properties, electrical conductivity, and cell alignment. For instance, engineered cardiac patches made from GelMA-CNT hydrogels could form 3D roll-shaped actuators that beat under the action of a periodic electrical stimulus, and even displaced autonomously in response to the frequency (Figure 5C) [71].

GelMA hydrogels, along with microfluidics, have also been used to develop and study cardiovascular-like tissues [11, 60]. GelMA hydrogels provide a benign microenvironment for the cells to bind and proliferate, while the microfluidic devices provide conditions that 
mimic the natural vascular environment in terms of a continuous supply of medium and a fluid shear stress effect on the cells. For instance, Annabi et al. found that cardiomyocytes (CMs) showed cellular alignment within the microchannels coated with GelMA hydrogels, driven by the flow direction. The CMs were able to create cardiac fiber-like tissues and developed the capability for spontaneous beating [60]. Chen et al. used a different microfluidic setting and found that shear stress induced by the medium flow had a significant effect on cell-cell interactions. The combined effect of the presence of the valvular endothelial cells and the shear stress induced by the flow hindered the pathological differentiation of VICs into $\alpha$-smooth muscle actin-positive myofibroblast [11].

Progresses have been made in the development of GelMA based scaffolds for regeneration of load-bearing tissues such as skeletal muscle, bone, and cartilage. An important challenge in designing scaffolds for these types of tissues is to achieve a set of mechanical properties that closely mimic those observed in native tissues. As discussed in previous sections, several successful strategies have been reported to reinforce GelMA by combining it with other components such as CNTs, GO, inorganic nanoparticles, other biopolymers, and synthetic polymers.

Mineralization is a particularly important property for bone tissue engineering and is needed to promote osteo-conduction. Recent reports demonstrated promising applications of GelMA hydrogels for the fabrication of functional bone scaffolds (Figure 5E) [78, 81, 82]. Zuo et al. also used GelMA hydrogels to build multiple-compartment, osteon-like structures with interconnected channels, aiming to resemble the haversian canal in native bone tissues. 
In vitro tests showed that human osteosarcoma cells (MG63) and HUVEC cells, growing in different compartments of the constructs, expressed osteogenic and vasculogenic genes, respectively [101]. Heo et al. reported the use of a hybrid material based on GelMA and gold nanoparticles to regenerate bone tissues [78].

GelMA hydrogels, and particularly hybrid hydrogels based on GelMA, have been studied as promising materials for cartilage tissue engineering. For example Klein's group explored the use of GelMA-HA scaffolds to repair cartilage tissues (Figure 3F) [15, 85, 102]. Interestingly, addition of HA derivatives changed the morphology of the chondrocytes and promoted chondrogenesis and the synthesis of ECM components (collagen type I and II and fibronectin) within the GelMA-HA scaffold [85]. A recent paper by Visser et al. showed that the reinforcement of GelMA hydrogels using 3D-printed microfibers (by a technique referred as melt electrospinning writing) produced gel-scaffold composites with a synergistically enhanced stiffness and elasticity similar to that of cartilage tissue [103].

Liver has a particular microarchitecture consisting of hexagonal units (lobules) assembled together. Zamanian et al. aimed to mimic this structure to engineer fully functional tissues and developed a method to fabricate cell-laden microgels that were capable of self-assembly into tissue-like structures by geometric control [53]. The use of GelMA microgels coupled with this assembling method confirmed that HepG2 cells co-cultured with $3 \mathrm{~T} 3$ fibroblasts were able to interact at the border of the microgels (Figure 5D). This effect could be mainly attributed to the cell-responsive nature of GelMA, since it allows cell elongation and migration. The ability for finely control of the microarchitecture of the 
scaffold and the feasibility for co-culturing cells in individual but communicating compartments open up great possibilities in the engineering of fully functional tissues.

GelMA hydrogels are also promising materials for engineering skin. In a recent contribution, Zhao et al. have shown that GelMA scaffolds can support the growth, differentiation, and stratification of keratinocytes into a functional multilayered epidermis-type tissue [104]. These results suggest that keratinocyte-laden GelMA hydrogels can be used in various epidermal tissue engineering applications, including wound dressing applications, to fabricate epidermal substitutes. They will also be useful as substrates for the construction of various in vitro skin models.

\subsection{Other applications of GelMA hydrogel}

The versatility of GelMA hydrogels allows for many interesting applications, other than those directly related to tissue engineering. For instance, GelMA hydrogels have been recently used as an alternative 3D culture system for cancer cells. Kaemmerer et al. reported that human epithelial ovarian cancer cells encapsulated in GelMA hydrogels showed high level metabolic rates and spheroid formation [16]. Control experiments with inhibition of MMPs revealed the role of MMP-sensitive sites in the promotion of cancer cell proliferation. Moreover, the in vitro cultured cancer cell spheroids demonstrated in vivo activity towards tumor development and formation in a mice model, which could be suppressed by anti-cancer drug treatment. The authors suggested that GelMA hydrogels could offer a cost-efficient system for 3D cancer cell culture [16].

Controlled drug release is another area where the use of GelMA hydrogels has been 
explored. A recent paper by Serafim et al described the evaluation of a family of GelMA-polyacrylamide (PAA) hybrid hydrogels as a matrix for the controlled release of a model drug, sodium nafcillin monohydrate, a $\beta$-lactam antibiotic frequently prescribed against staphylococci [17]. The authors demonstrated the possibility of tuning the release profile of the antibiotic by varying the composition of the hybrid (i.e., increasing the fraction of PAA). While pristine GelMA hydrogels released only $50 \%$ of the antibiotic in 10 hours, PAA-GelMA hybrids (1.6:1) released $95 \%$ of it in the same period of time. Another use of GelMA hydrogels in controlled release applications was introduced by Jeon et al., who dispersed drug-laden GelMA hydrogel droplets into an hMSC-laden bulk matrix composed of oxidized methacrylated alginate (OMA) [105]. This work offers proof-of-principle for sustained molecule delivery to encapsulated cells for different biomedical applications, such as immunomodulation and stem cell therapeutic applications, among others.

Other strategies have been devised to achieve a precise drug control release using GelMA hydrogel. For example, dielectrophoresis (DEP) techniques can be used to engineer accurate 3D concentration gradients of micro and nanoparticles loaded with drugs (or other compounds) in GelMA hydrogels (Figure 6A) [77]. The nanoparticle concentration gradient and the degree of crosslinking of the hydrogels offer the possibility of customizing programmed release profiles. Alternatively, cell-laden GelMA hydrogels engineered with 3D gradients can be used as research tools to study the effect of chemical gradients in cell systems. Recently, the use of a cell-laden GelMA microarray system for the massive screening of proper osteogenic differentiation conditions of mesenchymal stem cells was 
demonstrated [106].

GelMA hydrogels can also be used, if properly functionalized, for non-viral gene delivery applications. For example, GelMA hydrogels loaded with GO nanosheets functionalized with PEI were used as effective vehicles for transfection of endothelial cells and myocardial tissues in a rat model [75]. GelMA has also found application niches in fundamental research. For example, it has been used to devise chips for the capture, culture, and study of a single neuron (Figure 6B) [40] and to build 3D microfluidic systems (or components of them) for cell culture applications [38, 107]. Recently, Topkaya et al. used GelMA hydrogels to fabricate a proof-of-principle electrochemical sensor for DNA hybridization (Figure 6C) [108].

\section{Conclusions and Outlook}

We have reviewed several important aspects of GelMA-based hydrogel systems for biomedical applications. GelMA is developed from a natural polymer gelatin via one-step chemical modification. The introduction of photocrosslinkable methacryloyl substitution groups enables convenient and fast gelation upon exposure to light irradiation at the presence of photoinitiators. Many physical parameters of GelMA hydrogels, such as mechanical properties, pore sizes, degradation rates, and swell ratio can be readily tailored by changing the degree of methacryloyl substitution, concentration of the GelMA prepolymer, initiator concentration, and UV exposure time. Moreover, the resulting GelMA hydrogels retain the excellent biocompatibility and bioactivity of gelatin, such as promoting adhesion, spreading, and proliferation of various cell lines, due to the existence of cell adhesive RGD motifs and 
MMP-degradable amino acid sequences. Notably, this photocrosslinkable biomaterial allows construction of engineered cell-laden microconstructs with defined 3D architecture and topology via a panel of different microfabrication techniques, such as photopatterning, micromolding, and bioprinting. The 3D cell-laden constructs based on GelMA hydrogels could be designed to mimic the structure of native tissues, and thus promises their applications in tissue engineering and regenerative medicine. Another important field is to design hybrid hydrogels by mixing GelMA with other materials, such as inorganic particles, carbon materials, biopolymers, and synthetic polymers. This approach could generate hybrid materials that combine the advantageous properties of the other components, such as superior mechanical properties and conductivity, with the bioactivity of GelMA. GelMA based biomaterials will continue to serve as promising candidates in many other biomedical applications that remain to be explored.

In the near future, novel microfabrication techniques will further expand the spectrum of applications of GelMA derived scaffolds. Challenges remain at both extremes of the length-scales that are relevant to tissue engineering. The facsimile fabrication of hollow micro-capillaries remains to be fully addressed. The engineering approaches to produce vessels with lumens in the microscale have yet to be improved. The combined use of sacrificial filaments and microfluidics (or bio-printing) appears to be a part of the solution. In the other side of the spectrum of sizes, the construction of functional organoids and complex 3D tissues has to overcome oxygen transport limitations associated with length-scales in the order of centimeters. Here, self-assembly might become a key resource. The design of smart 
GelMA-based hydrogels_capable of sealing (and even healing) surgical injuries [109], releasing oxygen (among other nutrients), or capturing and removing inhibitory cell by-products—is a future direction for GelMA studies.

\section{Acknowledgements}

N.A. acknowledges the support from the National Health and Medical Research Council. The authors acknowledge funding from the National Science Foundation (EFRI-1240443), IMMODGEL (602694), and the National Institutes of Health (EB012597, AR057837, DE021468, HL099073, AI105024, AR063745). MMA gratefully acknowledge the institutional funding received from Tecnológico de Monterrey (seed funding to Strategic Research Groups, 2015) and funding provided from the CONACyT (Consejo Nacional de Ciencia y Tecnología, México) through Sistema Nacional de Investigadores (SNI). MMA, GTdS and A.K. acknowledge funding from MIT International Science and Technology Initiatives (MISTI). GTdS acknowledges funding form Fundación México en Harvard. 


\section{References:}

[1] Annabi N, Tamayol A, Uquillas JA, Akbari M, Bertassoni LE, Cha C, et al. 25th Anniversary Article: Rational Design and Applications of Hydrogels in Regenerative Medicine. Advanced Materials. 2014;26:85-124.

[2] Thiele J, Ma Y, Bruekers SMC, Ma S, Huck WTS. 25th Anniversary Article: Designer Hydrogels for Cell Cultures: A Materials Selection Guide. Advanced Materials. 2014;26:125-48.

[3] Alge DL, Anseth KS. Bioactive hydrogels: Lighting the way. Nature Materials. 2013;12:950-2.

[4] Luo Y, Shoichet MS. A photolabile hydrogel for guided three-dimensional cell growth and migration. Nature Materials. 2004;3:249-53.

[5] West JL. Protein-patterned hydrogels: Customized cell microenvironments. Nature Materials. 2011;10:727-9.

[6] Nichol JW, Koshy ST, Bae H, Hwang CM, Yamanlar S, Khademhosseini A. Cell-laden microengineered gelatin methacrylate hydrogels. Biomaterials. 2010;31:5536-44.

[7] Hutson CB, Nichol JW, Aubin H, Bae H, Yamanlar S, Al-Haque S, et al. Synthesis and Characterization of Tunable Poly(Ethylene Glycol): Gelatin Methacrylate Composite Hydrogels. Tissue Engineering Part A. 2011;17:1713-23.

[8] Nikkhah M, Eshak N, Zorlutuna P, Annabi N, Castello M, Kim K, et al. Directed endothelial cell morphogenesis in micropatterned gelatin methacrylate hydrogels. Biomaterials. 2012;33:9009-18.

[9] Chen Y-C, Lin R-Z, Qi H, Yang Y, Bae H, Melero-Martin JM, et al. Functional Human Vascular Network Generated in Photocrosslinkable Gelatin Methacrylate Hydrogels. Advanced Functional Materials. 2012;22:2027-39.

[10] Hosseini V, Ahadian S, Ostrovidov S, Camci-Unal G, Chen S, Kaji H, et al. Engineered Contractile Skeletal Muscle Tissue on a Microgrooved Methacrylated Gelatin Substrate. Tissue Engineering Part A. 2012;18:2453-65.

[11] Chen MB, Srigunapalan S, Wheeler AR, Simmons CA. A 3D microfluidic platform incorporating methacrylated gelatin hydrogels to study physiological cardiovascular cell-cell interactions. Lab on a Chip. 2013;13:2591-8.

[12] Bertassoni LE, Cardoso JC, Manoharan V, Cristino AL, Bhise NS, Araujo WA, et al. Direct-write bioprinting of cell-laden methacrylated gelatin hydrogels. Biofabrication. 2014;6:024105.

[13] Lin R-Z, Chen Y-C, Moreno-Luna R, Khademhosseini A, Melero-Martin JM. Transdermal regulation of vascular network bioengineering using a photopolymerizable methacrylated gelatin hydrogel. Biomaterials. 2013;34:6785-96.

[14] Van Den Bulcke AI, Bogdanov B, De Rooze N, Schacht EH, Cornelissen M, Berghmans H. Structural and Rheological Properties of Methacrylamide Modified Gelatin Hydrogels. Biomacromolecules. 2000;1:31-8.

[15] Schuurman W, Levett PA, Pot MW, van Weeren PR, Dhert WJA, Hutmacher DW, et al. Gelatin-Methacrylamide Hydrogels as Potential Biomaterials for Fabrication of 
Tissue-Engineered Cartilage Constructs. Macromolecular Bioscience. 2013;13:551-61.

[16] Kaemmerer E, Melchels FPW, Holzapfel BM, Meckel T, Hutmacher DW, Loessner D. Gelatine methacrylamide-based hydrogels: An alternative three-dimensional cancer cell culture system. Acta Biomaterialia. 2014;10:2551-62.

[17] Serafim A, Tucureanu C, Petre D-G, Dragusin D-M, Salageanu A, Van Vlierberghe S, et al. One-pot synthesis of superabsorbent hybrid hydrogels based on methacrylamide gelatin and polyacrylamide. Effortless control of hydrogel properties through composition design. New Journal of Chemistry. 2014;38:3112-26.

[18] Billiet T, Gevaert E, De Schryver T, Cornelissen M, Dubruel P. The 3D printing of gelatin methacrylamide cell-laden tissue-engineered constructs with high cell viability. Biomaterials. 2014;35:49-62.

[19] Liu Y, Chan-Park MB. A biomimetic hydrogel based on methacrylated dextran-graft-lysine and gelatin for 3D smooth muscle cell culture. Biomaterials. 2010;31:1158-70.

[20] Van den Steen PE, Dubois B, Nelissen I, Rudd PM, Dwek RA, Opdenakker G. Biochemistry and Molecular Biology of Gelatinase B or Matrix Metalloproteinase-9 (MMP-9). Critical Reviews in Biochemistry and Molecular Biology. 2002;37:375-536.

[21] Maurer PH. II. ANTIGENICITY OF GELATIN IN RABBITS AND OTHER SPECIES. The Journal of Experimental Medicine. 1954;100:515-23.

[22] Gorgieva S, Kokol V. Collagen-vs. gelatine-based biomaterials and their biocompatibility: review and perspectives: INTECH Open Access Publisher; 2011.

[23] Djabourov M, Papon P. Influence of thermal treatments on the structure and stability of gelatin gels. Polymer. 1983;24:537-42.

[24] Jayakrishnan A, Jameela SR. Glutaraldehyde as a fixative in bioprostheses and drug delivery matrices. Biomaterials. 1996;17:471-84.

[25] Olde Damink LHH, Dijkstra PJ, Van Luyn MJA, Van Wachem PB, Nieuwenhuis P, Feijen J. Crosslinking of dermal sheep collagen using hexamethylene diisocyanate. J Mater Sci: Mater Med. 1995;6:429-34.

[26] Sung H-W, Hsu H-L, Shih C-C, Lin D-S. Cross-linking characteristics of biological tissues fixed with monofunctional or multifunctional epoxy compounds. Biomaterials. 1996;17:1405-10.

[27] Petite H, Rault I, Huc A, Menasche P, Herbage D. Use of the acyl azide method for cross-linking collagen-rich tissues such as pericardium. Journal of Biomedical Materials Research. 1990;24:179-87.

[28] Aubin H, Nichol JW, Hutson CB, Bae H, Sieminski AL, Cropek DM, et al. Directed $3 \mathrm{D}$ cell alignment and elongation in microengineered hydrogels. Biomaterials. 2010;31:6941-51.

[29] Benton JA, DeForest CA, Vivekanandan V, Anseth KS. Photocrosslinking of Gelatin Macromers to Synthesize Porous Hydrogels That Promote Valvular Interstitial Cell Function. Tissue Engineering Part A. 2009;15:3221-30.

[30] Xu F, Inci F, Mullick O, Gurkan UA, Sung Y, Kavaz D, et al. Release of Magnetic Nanoparticles from Cell-Encapsulating Biodegradable Nanobiomaterials. ACS Nano. 
2012;6:6640-9.

[31] Xiao W, He J, Nichol JW, Wang L, Hutson CB, Wang B, et al. Synthesis and characterization of photocrosslinkable gelatin and silk fibroin interpenetrating polymer network hydrogels. Acta Biomaterialia. 2011;7:2384-93.

[32] Hoch E, Schuh C, Hirth T, Tovar GEM, Borchers K. Stiff gelatin hydrogels can be photo-chemically synthesized from low viscous gelatin solutions using molecularly functionalized gelatin with a high degree of methacrylation. Journal of Materials Science-Materials in Medicine. 2012;23:2607-17.

[33] Fairbanks BD, Schwartz MP, Bowman CN, Anseth KS. Photoinitiated polymerization of PEG-diacrylate with lithium phenyl-2,4,6-trimethylbenzoylphosphinate: polymerization rate and cytocompatibility. Biomaterials. 2009;30:6702-7.

[34] Benton JA, Fairbanks BD, Anseth KS. Characterization of valvular interstitial cell function in three dimensional matrix metalloproteinase degradable PEG hydrogels. Biomaterials. 2009;30:6593-603.

[35] Koshy ST, Ferrante TC, Lewin SA, Mooney DJ. Injectable, porous, and cell-responsive gelatin cryogels. Biomaterials. 2014;35:2477-87.

[36] Van Vlierberghe S, Cnudde V, Dubruel P, Masschaele B, Cosijns A, De Paepe I, et al. Porous gelatin hydrogels: 1. Cryogenic formation and structure analysis. Biomacromolecules. 2007;8:331-7.

[37] Van Vlierberghe S, Dubruel P, Schacht E. Effect of Cryogenic Treatment on the Rheological Properties of Gelatin Hydrogels. Journal of Bioactive and Compatible Polymers. 2010;25:498-512.

[38] Lee Y, Lee JM, Bae P-K, Chung IY, Chung BH, Chung BG. Photo-crosslinkable hydrogel-based 3D microfluidic culture device. Electrophoresis. 2015;36:994-1001.

[39] Dubruel P, Unger R, Van Vlierberghe S, Cnudde V, Jacobs PJS, Schacht E, et al. Porous gelatin hydrogels: 2. In vitro cell interaction study. Biomacromolecules. 2007;8:338-44.

[40] Fan Y, Xu F, Huang G, Lu TJ, Xing W. Single neuron capture and axonal development in three-dimensional microscale hydrogels. Lab on a Chip. 2012;12:4724-31.

[41] Gauvin R, Chen Y-C, Lee JW, Soman P, Zorlutuna P, Nichol JW, et al. Microfabrication of complex porous tissue engineering scaffolds using 3D projection stereolithography. Biomaterials. 2012;33:3824-34.

[42] Soman P, Chung PH, Zhang AP, Chen S. Digital Microfabrication of User-Defined 3D Microstructures in Cell-Laden Hydrogels. Biotechnology and Bioengineering. 2013;110:3038-47.

[43] Grogan SP, Chung PH, Soman P, Chen P, Lotz MK, Chen S, et al. Digital micromirror device projection printing system for meniscus tissue engineering. Acta Biomaterialia. 2013;9:7218-26.

[44] Ovsianikov A, Deiwick A, Van Vlierberghe S, Dubruel P, Moeller L, Draeger G, et al. Laser Fabrication of Three-Dimensional CAD Scaffolds from Photosensitive Gelatin for Applications in Tissue Engineering. Biomacromolecules. 2011;12:851-8.

[45] Ovsianikov A, Deiwick A, Van Vlierberghe S, Pflaum M, Wilhelmi M, Dubruel P, et 
al. Laser Fabrication of 3D Gelatin Scaffolds for the Generation of Bioartificial Tissues. Materials. 2011;4:288-99.

[46] Ovsianikov A, Muehleder S, Torgersen J, Li Z, Qin X-H, Van Vlierberghe S, et al. Laser Photofabrication of Cell-Containing Hydrogel Constructs. Langmuir. 2014;30:3787-94.

[47] Qi H, Du Y, Wang L, Kaji H, Bae H, Khademhosseini A. Patterned Differentiation of Individual Embryoid Bodies in Spatially Organized 3D Hybrid Microgels. Advanced Materials. 2010;22:5276-81.

[48] Ahadian S, Ramon-Azcon J, Ostrovidov S, Camci-Unal G, Hosseini V, Kaji H, et al. Interdigitated array of $\mathrm{Pt}$ electrodes for electrical stimulation and engineering of aligned muscle tissue. Lab on a Chip. 2012;12:3491-503.

[49] Obregon R, Ahadian S, Ramon-Azcon J, Chen L, Fujita T, Shiku H, et al. Non-invasive measurement of glucose uptake of skeletal muscle tissue models using a glucose nanobiosensor. Biosensors \& Bioelectronics. 2013;50:194-201.

[50] Hosseini V, Kollmannsberger P, Ahadian S, Ostrovidov S, Kaji H, Vogel V, et al. Fiber-Assisted Molding (FAM) of Surfaces with Tunable Curvature to Guide Cell Alignment and Complex Tissue Architecture. Small. 2014;10:4851-7.

[51] Annabi N, Tsang K, Mithieux SM, Nikkhah M, Ameri A, Khademhosseini A, et al. Highly Elastic Micropatterned Hydrogel for Engineering Functional Cardiac Tissue. Advanced Functional Materials. 2013;23:4950-9.

[52] Sadr N, Zhu M, Osaki T, Kakegawa T, Yang Y, Moretti M, et al. SAM-based cell transfer to photopatterned hydrogels for microengineering vascular-like structures. Biomaterials. 2011;32:7479-90.

[53] Zamanian B, Masaeli M, Nichol JW, Khabiry M, Hancock MJ, Bae H, et al. Interface-Directed Self-Assembly of Cell-Laden Microgels. Small. 2010;6:937-44.

[54] Qi H, Ghodousi M, Du Y, Grun C, Bae H, Yin P, et al. DNA-directed self-assembly of shape-controlled hydrogels. Nature Communications. 2013;4:doi:10.1038/ncomms3275.

[55] Xu F, Wu C-aM, Rengarajan V, Finley TD, Keles HO, Sung Y, et al. Three-Dimensional Magnetic Assembly of Microscale Hydrogels. Advanced Materials. 2011;23:4254-60.

[56] Tasoglu S, Yu CH, Liaudanskaya V, Guven S, Migliaresi C, Demirci U. Magnetic Levitational Assembly for Living Material Fabrication. Advanced Healthcare Materials. 2015:doi:10.1002/adhm.201500092.

[57] Hancock MJ, Piraino F, Camci-Unal G, Rasponi M, Khademhosseini A. Anisotropic material synthesis by capillary flow in a fluid stripe. Biomaterials. 2011;32:6493-504.

[58] Piraino F, Camci-Unal G, Hancock MJ, Rasponi M, Khademhosseini A. Multi-gradient hydrogels produced layer by layer with capillary flow and crosslinking in open microchannels. Lab on a Chip. 2012;12:659-61.

[59] Hsieh H-Y, Camci-Unal G, Huang T-W, Liao R, Chen T-J, Paul A, et al. Gradient static-strain stimulation in a microfluidic chip for 3D cellular alignment. Lab on a Chip. 2014;14:482-93.

[60] Annabi N, Selimovic S, Acevedo Cox JP, Ribas J, Bakooshli MA, Heintze D, et al. Hydrogel-coated microfluidic channels for cardiomyocyte culture. Lab on a Chip. 
2013;13:3569-77.

[61] Hoch E, Hirth T, Tovar GEM, Borchers K. Chemical tailoring of gelatin to adjust its chemical and physical properties for functional bioprinting. Journal of Materials Chemistry B. 2013;1:5675-85.

[62] Melchels FPW, Dhert WJA, Hutmacher DW, Malda J. Development and characterisation of a new bioink for additive tissue manufacturing. Journal of Materials Chemistry B. 2014;2:2282-9.

[63] Bertassoni LE, Cecconi M, Manoharan V, Nikkhah M, Hjortnaes J, Cristino AL, et al. Hydrogel bioprinted microchannel networks for vascularization of tissue engineering constructs. Lab on a Chip. 2014;14:2202-11.

[64] Kolesky DB, Truby RL, Gladman AS, Busbee TA, Homan KA, Lewis JA. 3D Bioprinting of Vascularized, Heterogeneous Cell-Laden Tissue Constructs. Advanced Materials. 2014;26:3124-30.

[65] Tamayol A, Akbari M, Annabi N, Paul A, Khademhosseini A, Juncker D. Fiber-Based Tissue Engineering: Progress, Challenges, and Opportunities. Biotechnology Advances. 2013;31:669-87.

[66] Hwang CM, Khademhosseini A, Park Y, Sun K, Lee S-H. Microfluidic chip-based fabrication of PLGA microfiber scaffolds for tissue engineering. Langmuir. 2008;24:6845-51.

[67] Chung BG, Lee K-H, Khademhosseini A, Lee S-H. Microfluidic fabrication of microengineered hydrogels and their application in tissue engineering. Lab on a Chip. 2012;12:45-59.

[68] Akbari M, Tamayol A, Laforte V, Annabi N, Najafabadi AH, Khademhosseini A, et al. Composite Living Fibers for Creating Tissue Constructs Using Textile Techniques. Advanced Functional Materials. 2014;24:4060-7.

[69] Shi X, Ostrovidov S, Zhao Y, Liang X, Kasuya M, Kurihara K, et al. Microfluidic Spinning of Cell-Responsive Grooved Microfibers. Advanced Functional Materials. 2015;25:2250-9.

[70] Shin SR, Bae H, Cha JM, Mun JY, Chen Y-C, Tekin H, et al. Carbon Nanotube Reinforced Hybrid Microgels as Scaffold Materials for Cell Encapsulation. ACS Nano. 2012;6:362-72.

[71] Shin SR, Aghaei-Ghareh-Bolagh B, Dang TT, Topkaya SN, Gao X, Yang SY, et al. Cell-laden Microengineered and Mechanically Tunable Hybrid Hydrogels of Gelatin and Graphene Oxide. Advanced Materials. 2013;25:6385-91.

[72] Ramon-Azcon J, Ahadian S, Estili M, Liang X, Ostrovidov S, Kaji H, et al. Dielectrophoretically Aligned Carbon Nanotubes to Control Electrical and Mechanical Properties of Hydrogels to Fabricate Contractile Muscle Myofibers. Advanced Materials. 2013;25:4028-34.

[73] Ahadian S, Ramón-Azcón J, Estili M, Liang X, Ostrovidov S, Shiku H, et al. Hybrid hydrogels containing vertically aligned carbon nanotubes with anisotropic electrical conductivity for muscle myofiber fabrication. Scientific Reports. 2014;4:doi:10.1038/srep04271.

[74] Cha C, Shin SR, Gao X, Annabi N, Dokmeci MR, Tang X, et al. Controlling 
Mechanical Properties of Cell-Laden Hydrogels by Covalent Incorporation of Graphene Oxide. Small. 2014;10:514-23.

[75] Paul A, Hasan A, Al Kindi H, Gaharwar AK, Rao VTS, Nikkhah M, et al. Injectable Graphene Oxide/Hydrogel-Based Angiogenic Gene Delivery System for Vasculogenesis and Cardiac Repair. ACS Nano. 2014;8:8050-62.

[76] Ahadian S, Estili M, Surya VJ, Ramon-Azcon J, Liang X, Shiku H, et al. Facile and green production of aqueous graphene dispersions for biomedical applications. Nanoscale. 2015;7:6436-43.

[77] Ahadian S, Ramon-Azcon J, Estili M, Obregon R, Shiku H, Matsue T. Facile and rapid generation of 3D chemical gradients within hydrogels for high-throughput drug screening applications. Biosensors \& Bioelectronics. 2014;59:166-73.

[78] Heo DN, Ko W-K, Bae MS, Lee JB, Lee D-W, Byun W, et al. Enhanced bone regeneration with a gold nanoparticle-hydrogel complex. Journal of Materials Chemistry B. 2014;2:1584-93.

[79] Kang H, Shih Y-RV, Hwang Y, Wen C, Rao V, Seo T, et al. Mineralized gelatin methacrylate-based matrices induce osteogenic differentiation of human induced pluripotent stem cells. Acta Biomaterialia. 2014;10:4961-70.

[80] Cha C, Oh J, Kim K, Qiu Y, Joh M, Shin SR, et al. Microfluidics-Assisted Fabrication of Gelatin-Silica Core-Shell Microgels for Injectable Tissue Constructs. Biomacromolecules. 2014;15:283-90.

[81] Zhou L, Tan G, Tan Y, Wang H, Liao J, Ning C. Biomimetic mineralization of anionic gelatin hydrogels: effect of degree of methacrylation. RSC Advances. 2014;4:21997-2008.

[82] Tan G, Zhou L, Ning C, Tan Y, Ni G, Liao J, et al. Biomimetically-mineralized composite coatings on titanium functionalized with gelatin methacrylate hydrogels. Applied Surface Science. 2013;279:293-9.

[83] Xavier JR, Thakur T, Desai P, Jaiswal MK, Sears N, Cosgriff-Hernandez E, et al. Bioactive Nanoengineered Hydrogels for Bone Tissue Engineering: A Growth-Factor-Free Approach. ACS Nano. 2015;9:3109-18.

[84] Gaharwar AK, Mihaila SM, Swami A, Patel A, Sant S, Reis RL, et al. Bioactive Silicate Nanoplatelets for Osteogenic Differentiation of Human Mesenchymal Stem Cells. Advanced Materials. 2013;25:3329-36.

[85] Levett PA, Melchels FPW, Schrobback K, Hutmacher DW, Malda J, Klein TJ. A biomimetic extracellular matrix for cartilage tissue engineering centered on photocurable gelatin, hyaluronic acid and chondroitin sulfate. Acta Biomaterialia. 2014;10:214-23.

[86] Wang H, Zhou L, Liao J, Tan Y, Ouyang K, Ning C, et al. Cell-laden photocrosslinked GelMA-DexMA copolymer hydrogels with tunable mechanical properties for tissue engineering. Journal of Materials Science-Materials in Medicine. 2014;25:2173-83.

[87] Shin SR, Jung SM, Zalabany M, Kim K, Zorlutuna P, Kim SB, et al. Carbon-Nanotube-Embedded Hydrogel Sheets for Engineering Cardiac Constructs and Bioactuators. ACS Nano. 2013;7:2369-80.

[88] Camci-Unal G, Cuttica D, Annabi N, Demarchi D, Khademhosseini A. Synthesis and Characterization of Hybrid Hyaluronic Acid-Gelatin Hydrogels. Biomacromolecules. 
2013;14:1085-92.

[89] Hjortnaes J, Camci-Unal G, Hutcheson JD, Jung SM, Schoen FJ, Kluin J, et al. Directing Valvular Interstitial Cell Myofibroblast-Like Differentiation in a Hybrid Hydrogel Platform. Advanced Healthcare Materials. 2015;4:121-30.

[90] Shin H, Olsen BD, Khademhosseini A. The mechanical properties and cytotoxicity of cell-laden double-network hydrogels based on photocrosslinkable gelatin and gellan gum biomacromolecules. Biomaterials. 2012;33:3143-52.

[91] Bae H, Ahari AF, Shin H, Nichol JW, Hutson CB, Masaeli M, et al. Cell-laden microengineered pullulan methacrylate hydrogels promote cell proliferation and 3D cluster formation. Soft Matter. 2011;7:1903-11.

[92] Li Y, San BH, Kessler JL, Kim JH, Xu Q, Hanes J, et al. Non-Covalent Photo-Patterning of Gelatin Matrices Using Caged Collagen Mimetic Peptides. Macromolecular Bioscience. 2015;15:52-62.

[93] Visser J, Gawlitta D, Benders KEM, Toma SMH, Pouran B, van Weeren PR, et al. Endochondral bone formation in gelatin methacrylamide hydrogel with embedded cartilage-derived matrix particles. Biomaterials. 2015;37:174-82.

[94] Daniele MA, Adams AA, Naciri J, North SH, Ligler FS. Interpenetrating networks based on gelatin methacrylamide and PEG formed using concurrent thiol click chemistries for hydrogel tissue engineering scaffolds. Biomaterials. 2014;35:1845-56.

[95] Fu Y, Xu K, Zheng X, Giacomin AJ, Mix AW, Kao WJ. 3D cell entrapment in crosslinked thiolated gelatin-poly(ethylene glycol) diacrylate hydrogels. Biomaterials. 2012;33:48-58.

[96] Fathi A, Lee S, Breen A, Shirazi AN, Valtchev P, Dehghani F. Enhancing the mechanical properties and physical stability of biomimetic polymer hydrogels for micro-patterning and tissue engineering applications. European Polymer Journal. 2014;59:161-70.

[97] Nemeth CL, Janebodin K, Yuan AE, Dennis JE, Reyes M, Kim D-H. Enhanced Chondrogenic Differentiation of Dental Pulp Stem Cells Using Nanopatterned PEG-GelMA-HA Hydrogels. Tissue Engineering Part A. 2014;20:2817-29.

[98] Pedron S, Harley BAC. Impact of the biophysical features of a 3D gelatin microenvironment on glioblastoma malignancy. Journal of Biomedical Materials Research Part A. 2013;101:3404-15.

[99] Boere KWM, Visser J, Seyednejad H, Rahimian S, Gawlitta D, van Steenbergen MJ, et al. Covalent attachment of a three-dimensionally printed thermoplast to a gelatin hydrogel for mechanically enhanced cartilage constructs. Acta Biomaterialia. 2014;10:2602-11.

[100] Eslami M, Vrana NE, Zorlutuna P, Sant S, Jung S, Masoumi N, et al. Fiber-reinforced hydrogel scaffolds for heart valve tissue engineering. Journal of Biomaterials Applications. 2014;29:399-410.

[101] Zuo Y, Xiao W, Chen X, Tang Y, Luo H, Fan H. Bottom-up approach to build osteon-like structure by cell-laden photocrosslinkable hydrogel. Chemical Communications. 2012;48:3170-2.

[102] Levett PA, Melchels FPW, Schrobback K, Hutmacher DW, Malda J, Klein TJ. 
Chondrocyte redifferentiation and construct mechanical property development in single-component photocrosslinkable hydrogels. Journal of Biomedical Materials Research Part A. 2014;102:2544-53.

[103] Visser J, Melchels FPW, Jeon JE, van Bussel EM, Kimpton LS, Byrne HM, et al. Reinforcement of hydrogels using three-dimensionally printed microfibres. Nature Communications. 2015;6:doi:10.1038/ncomms7933.

[104] Zhao X, Lang Q, Yildirimer L, Lin ZY, Cui W, Annabi N, et al. Photocrosslinkable Gelatin Hydrogel for Epidermal Tissue Engineering. Advanced Healthcare Materials. 2015:doi: 10.1002/adhm.201500005.

[105] Jeon O, Wolfson DW, Alsberg E. In-Situ Formation of Growth-Factor-Loaded Coacervate Microparticle-Embedded Hydrogels for Directing Encapsulated Stem Cell Fate. Advanced Materials. 2015;27:2216-23.

[106] Dolatshahi-Pirouz A, Nikkhah M, Gaharwar AK, Hashmi B, Guermani E, Aliabadi $\mathrm{H}$, et al. A combinatorial cell-laden gel microarray for inducing osteogenic differentiation of human mesenchymal stem cells. Scientific Reports. 2014;4:doi:10.1038/srep03896.

[107] Rahim R, Manuel O, Amy D, Tejasvi P, Mehmet RD, Ali K, et al. A Janus-paper PDMS platform for air-liquid interface cell culture applications. Journal of Micromechanics and Microengineering. 2015;25:055015.

[108] Topkaya SN. Gelatin methacrylate (GelMA) mediated electrochemical DNA biosensor for DNA hybridization. Biosensors and Bioelectronics. 2015;64:456-61.

[109] Kazemzadeh-Narbat M, Annabi N, Khademhosseini A. Surgical sealants and high strength adhesives. Materials Today. 2015;18:176-7. 

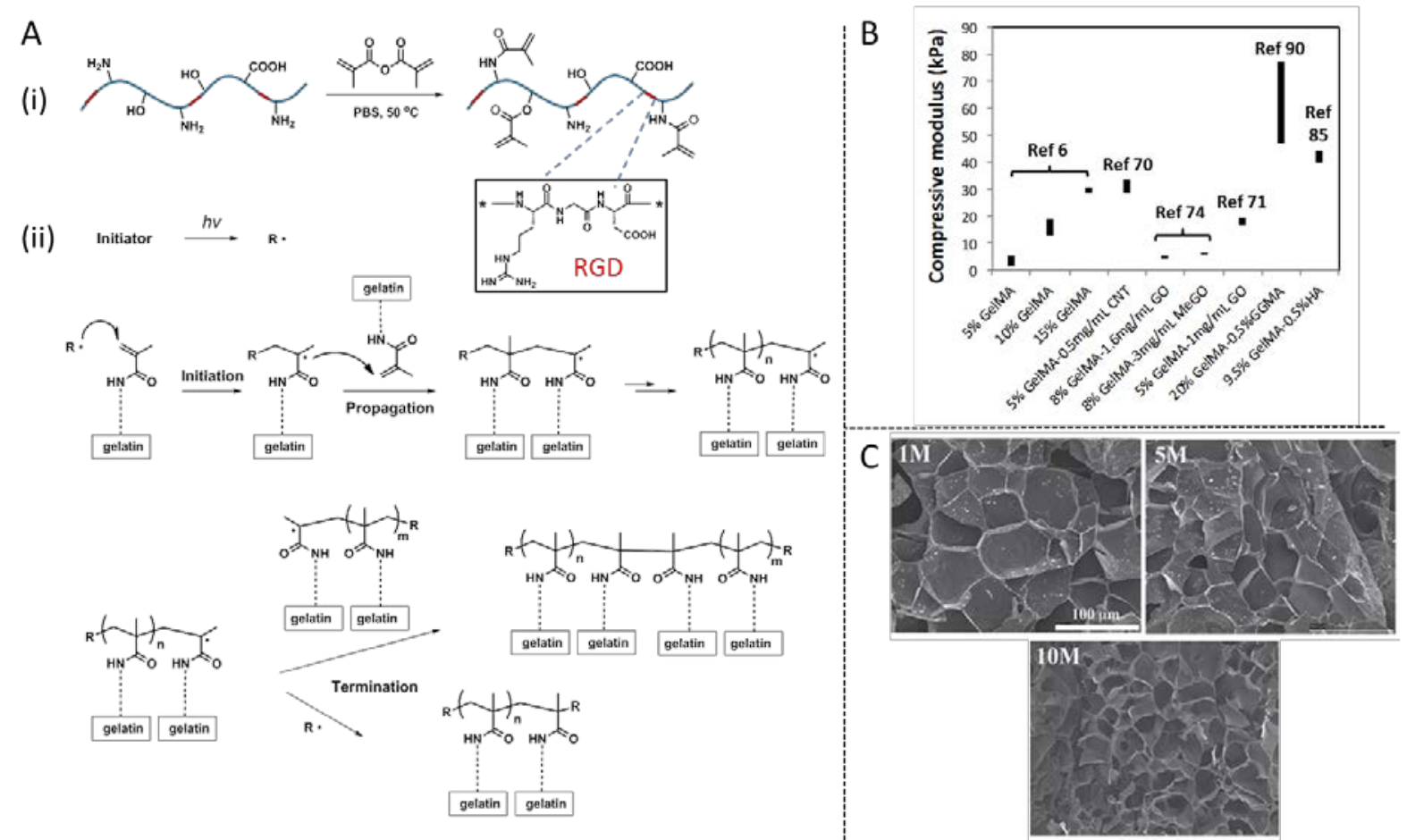

Figure 1. Synthesis and characterization of GelMA hydrogels. (A) Scheme for preparation of photocrosslinked GelMA hydrogel. (i) Reaction of gelatin and methacrylic anhydride for grafting of methacryloyl substitution groups. The modification occurs at primary amine and hydroxyl groups. The RGD domains are illustrated as red segments along the GelMA chains, and their chemical structure is depicted within the inset. (ii) Representative reactions during the photocrosslinking of GelMA to form hydrogel networks. Free radicals are generated from photoinitiators, which initiate the chain polymerization of the methacryloyl substitutions. Propagation occurs between methacryloyl groups located on the same chain and on different chains. Termination occurs between two propagating chains or between one propagating chain and a second radical. Chain transfers and many other minor reactions are not shown, for clarity. (B) The compressive modulus reported by several studies on GelMA hydrogels [6, $70,71,74,85,90]$. (C) SEM images of GelMA hydrogels, showing the effect of the degree of 
methacryloyl substitution on the pore sizes of GelMA hydrogels. Adapted from Chen et al. [9], with permission from Wiley, copyright 2012. 

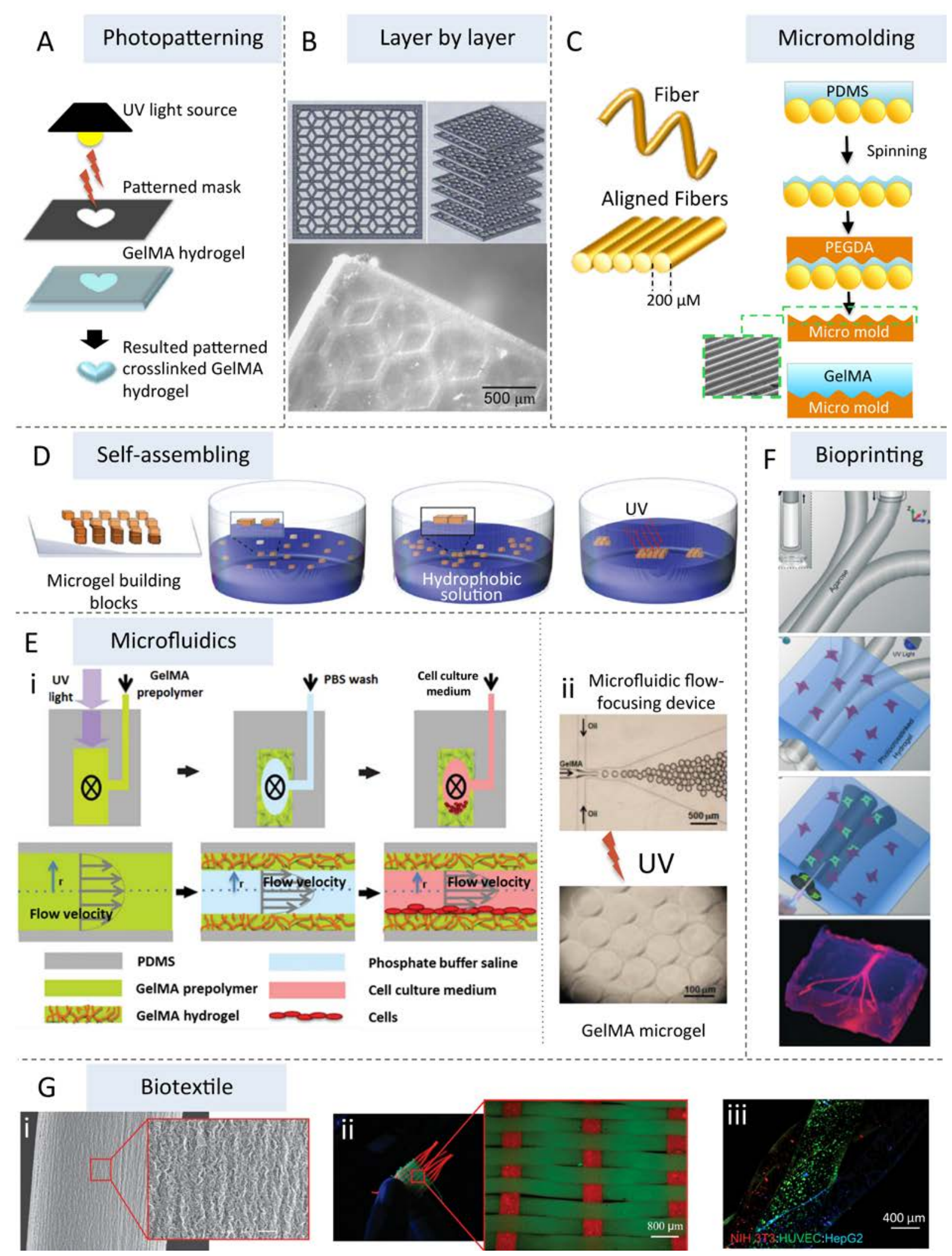

Figure 2. Microfabrication techniques used to produce GelMA hydrogels constructs. (A)

Schematic representation of photopatterning of GelMA using a pre-patterned photomask (B)

Stacked layers of patterned GelMA hydrogels fabricated using a micro-mirror projection stereolithography system. Adapted from Gauvin et al. [41], with permission from Elsevier, 
copyright 2012. (C) Schematic representation of a fiber-assisted micromolding technique for production of parallel microgrooved surfaces that serve as a template for micropatterning GelMA. Adapted from Hosseini et al. [1], with permission from Wiley, copyright 2014. (D) Schematic representation of the self-assembly of microgels fabricated by photopatterning. Adapted from Zamanian et al. [53], with permission from Wiley, copyright 2010. (E) Examples of the microfluidics of GelMA hydrogels microfabrication. (i) Schematic representation of the method for coating microchannels with GelMA hydrogel. Adapted from Annabi et al. [60], with permission from The Royal Society of Chemistry, copyright 2013. (ii) Fabrication of spherical GelMA microhydrogels using a microfluidic flow-focusing device. Adapted from Cha et al. [80], with permission from the American Chemical Society, copyright 2014. (F) Schematic representation of the bioprinting method for fabricating microchannels inside a GelMA hydrogel using an agarose template. Adapted from Bertassoni et al. [63], with permission from The Royal Society of Chemistry, copyright 2014. (G) Biotextile techniques as applied to the microfabrication of hybrid alginate and GelMA fibers and their assembly; (i) a representative SEM image of fabricated fiber; (ii) picture and micrograph of a typical woven fabric; (iii) a braided construct from NIH 3T3 fibroblasts, HUVECs, and hepatocytes (HepG2) as a liver model, with permission from the Wiley, copyright 2015. 

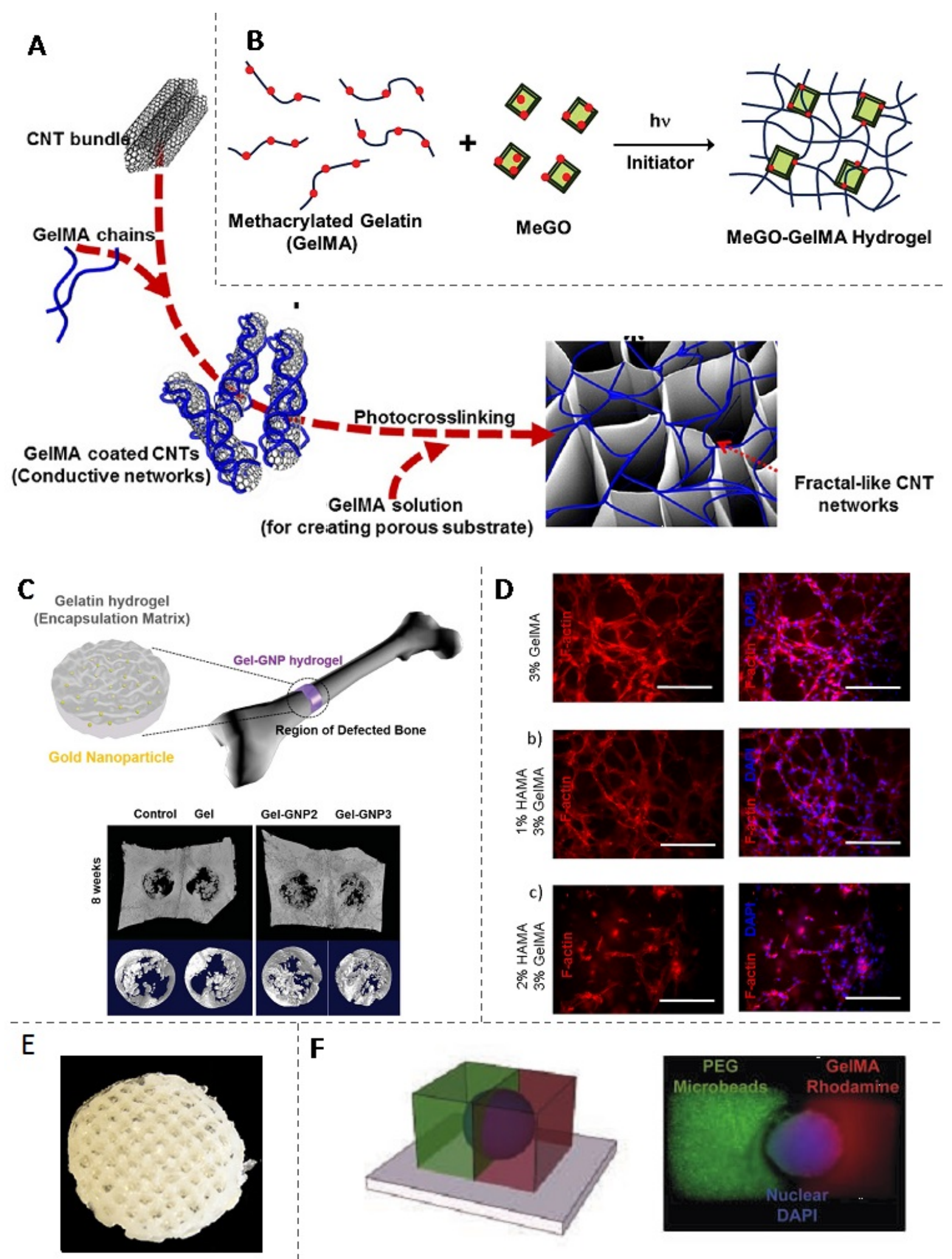

Figure 3. Examples GelMA-based hybrid hydrogels. (A) Schematic illustration of the preparation of GelMA-carbon nanotube (CNT) hybrids. GelMA coated carbon nanotubes are mixed with GelMA prepolymer and then cross-linked using UV to develop porous structures with improved mechanical and conductive productivities. Adapted from Shin et al. [87] with 
permission from The American Chemical Society, copyright 2013. (B) Scheme of the preparation of GelMA-methacrylated graphene oxide (MeGO) hybrid. Red points indicate the photocrosslinkable methacryloyl substitution groups. Adapted from Cha et al. [74] with permission from Wiley, copyright 2014. (C) Cartoon illustration of the GelMA-gold nanoparticles (GNP) hybrid and representation of its application as scaffolds for bone regeneration. The addition of gold nanoparticles enhances bone recovery in an animal model. After 8 weeks, bone defects induced in rabbits recovered sooner when treated with GelMA hydrogels with 0.071 and $0.20 \mu \mathrm{g} / \mathrm{mL}$ of gold nanoparticles (Gel-GNP2 and GelGNP3 respectively) than with pristine GelMA hydrogels (Gel). Adapted from Heo et al. [78] with permission from The Royal Society of Chemistry, copyright 2014. (D) Cytoskeleton and nuclei staining (F-actin/DAPI) for HUVEC-laden HAMA-GelMA hybrid hydrogels in 3D. By changing the concentration of HAMA and GelMA, it is possible to fabricate hybrid hydrogels with different stiffness that allows for tunable cellular response. Cell spreading images are taken at day 7. Scale bars represent $100 \mu \mathrm{m}$. Adapted from Camci-Unal et al. [88] with permission from ACS publications, copyright 2013. (E) Hybrid construct made with GelMA added to a framework made with methacrylated poly(hydroxymethylglycolide-co-e-caprolactone) and poly-e-caprolactone. Adapted from Boere et al. [99] with permission from Elsevier, copyright 2014. (F) Embryoid bodies encapsulated in GelMA/PEG hybrid microgels. Adapted from Qi et al. [47] with permission from Wiley, copyright 2010. 


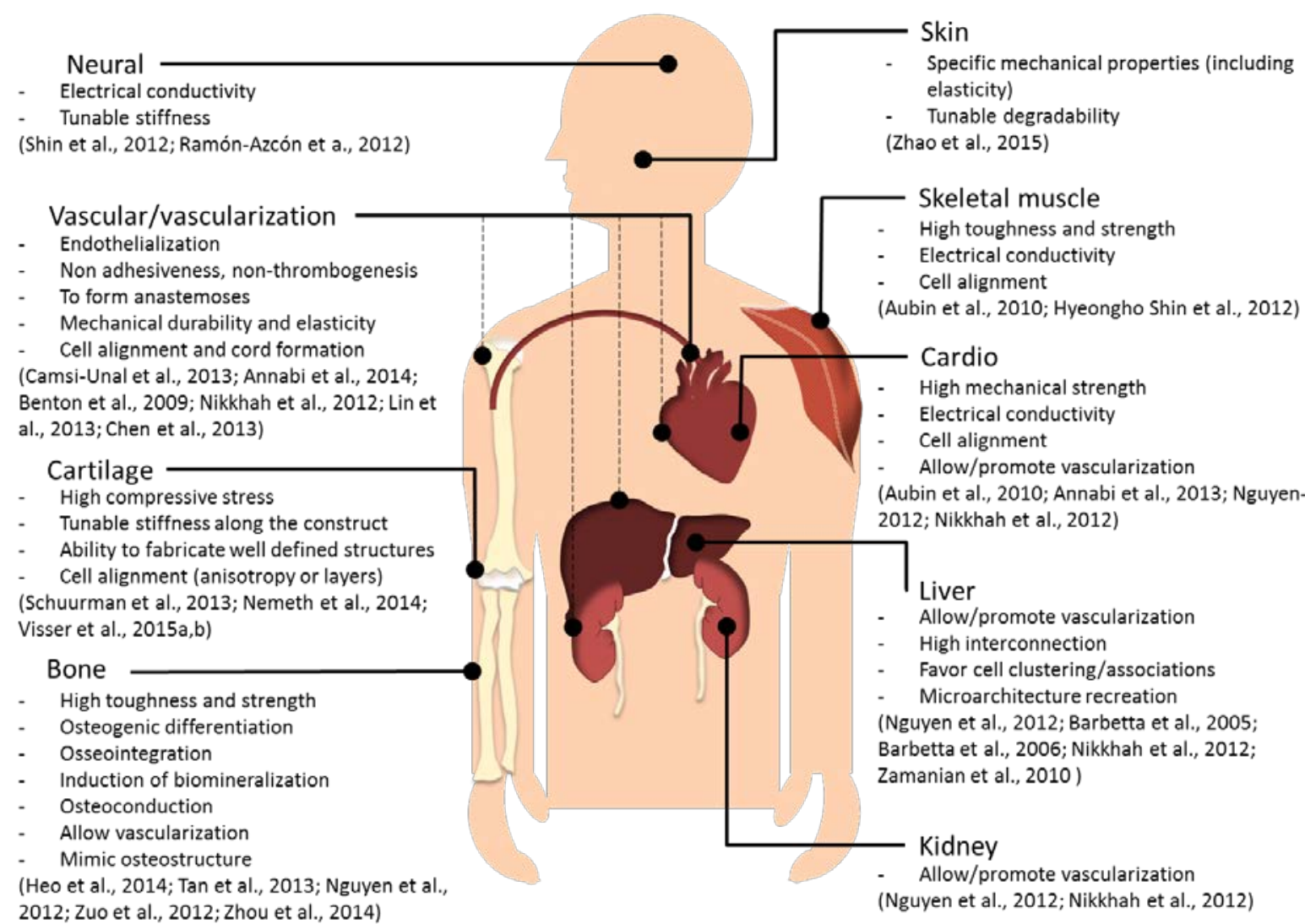

Figure 4. The specific characteristics and functions of each tissue impose particular tissue engineering requirements. In recent literature reports, GelMA hydrogels have proven to be a flexible and highly tunable platform for diverse tissue engineering applications in the areas of neural, cardiovascular, cartilage, bone, muscle, liver, and kidney engineering. 


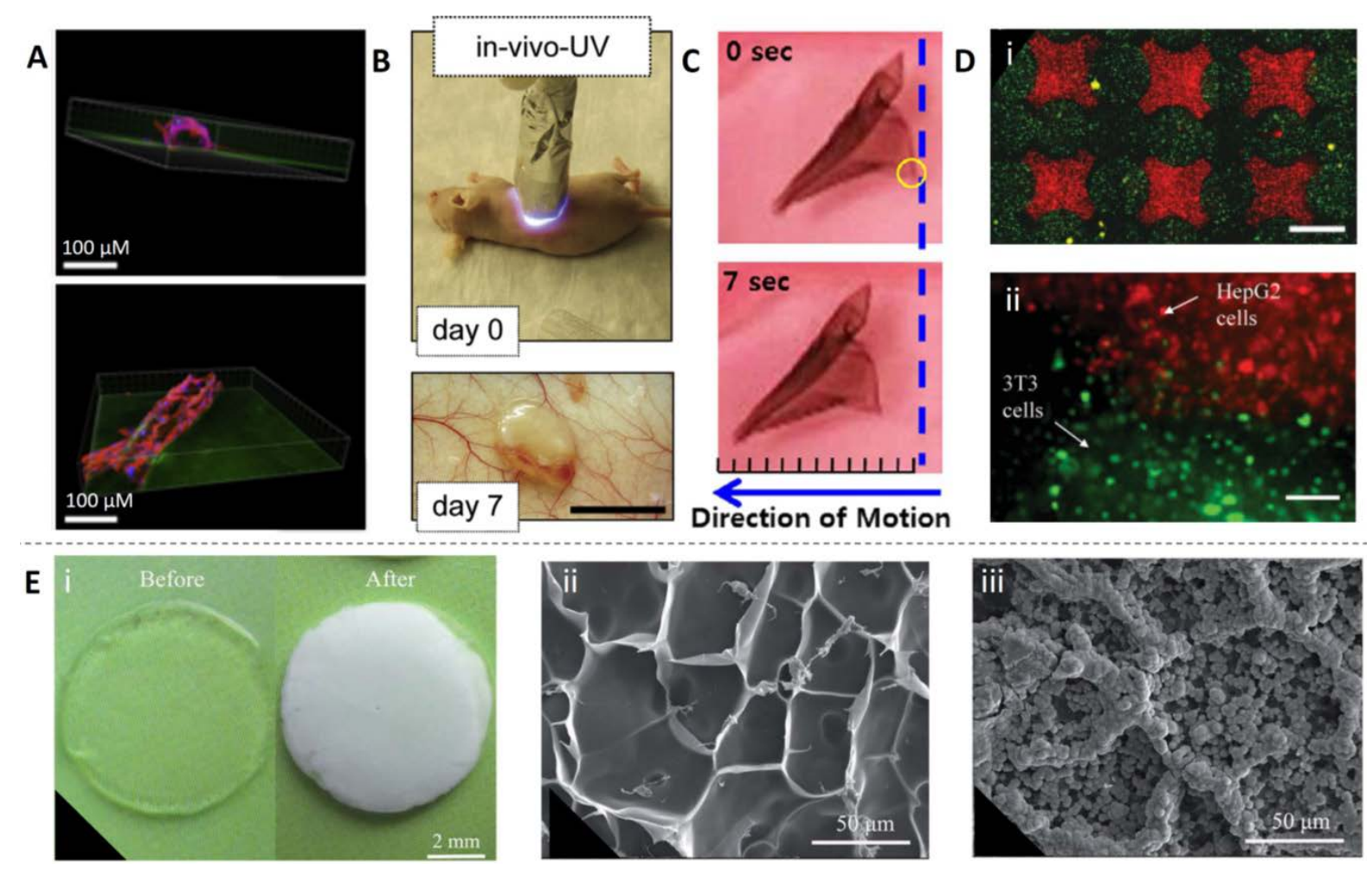

Figure 5. Representative examples of applications of GelMA hydrogels in tissue engineering.

(A) Confocal images of 3D cord formation of HUVEC cells on GelMA micropatterned constructs (scale bar: $100 \mu \mathrm{m}$ ). Adapted from Nikkhah et al. [8] with permission from Elsevier, copyright 2012. (B) Transdermal polymerization of GelMA in vivo, and the subcutaneous scaffold after 7 days showing vascularization (scale bar: $1 \mathrm{~cm}$ ). Adapted from Lin et al. [13] with permission from Elsevier, copyright 2013. (C) Engineered triangular swimmers made of GelMA-CNT showing spontaneous linear traveling (Each ruler marker is $1 \mathrm{~mm})$. Adapted from Shin et al. [87] with permission from The American Chemical Society, copyright 2013. (D) Fluorescence images of assembly of microgels structures with co-culture of 3 T3 fibroblasts and HepG2 cells (scale bar: $100 \mu \mathrm{m}$ ). Adapted from Zamanian et al. [53] with permission from Wiley, copyright 2010. (E) (i) GelMA scaffolds before and after 12 days of mineralization (scale bar: $2 \mathrm{~mm}$ ); (ii) SEM images of GelMA hydrogels before and (iii) after mineralization (scale bar: $50 \mu \mathrm{m}$ ). Adapted from Zhou et al. [81] with permission from The Royal Society of Chemistry, copyright 2014. 
A
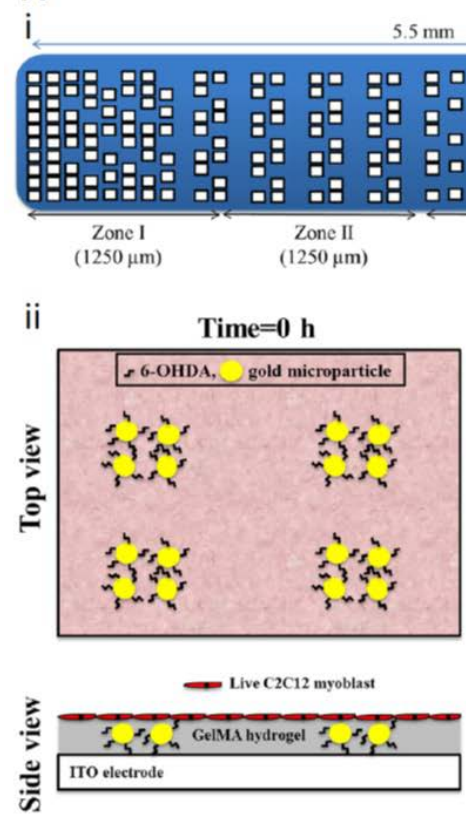

B

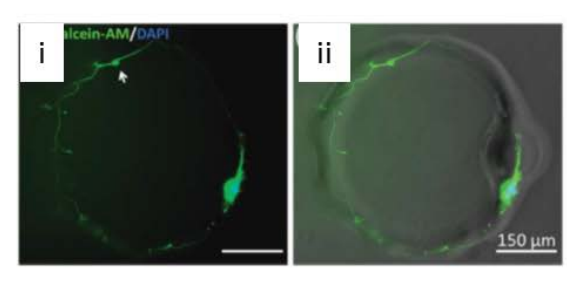

Time $=24 \mathrm{~h}$

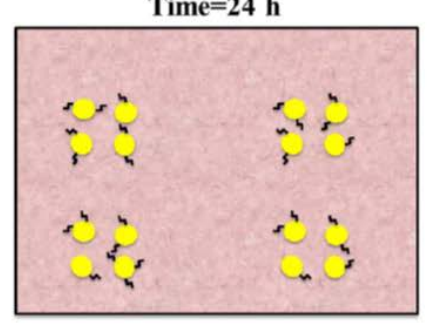

- Dead C2C12 myoblas

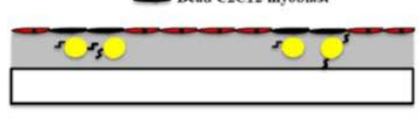

C

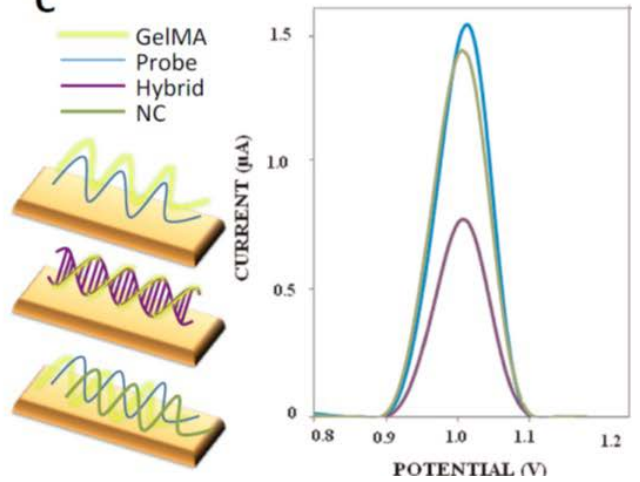

Figure 6. Examples of applications of GelMA hydrogels other than in tissue engineering. (A)

A gradient system made with GelMA hydrogels using dielectrophoresis. (i) Example of a device with four different zones of gradients; (ii) representation of a method to evaluate the viability of cells (C2C12 myoblasts) when cultured on GelMA gradient hydrogels containing a drug (6-hydroxydopamine: 6-OHDA) immobilized on gold microparticles. Adapted from Ahadian et al. [77] with permission from Elsevier, copyright 2014. (B) GelMA hydrogel ring as a device for the capture, culture, and study of a single neuron cell. (i) Calcein-AM/DAPI stained image and (ii) merged image of the single neuron cell encapsulated in the assembled GelMA hydrogel loop (scale bar: $150 \mu \mathrm{m}$ ). Adapted from Fan et al. [40] with permission from The Royal Society of Chemistry, copyright 2012. (C) Illustration of the use of GelMA in an electrochemical biosensor to detect DNA hybridization. Adapted from Topkaya et al. [108] with permission from Elsevier, copyright 2015. 
Table 1. Examples of hybrid hydrogels based on GelMA.

\begin{tabular}{|c|c|c|c|c|c|}
\hline Category & $\begin{array}{l}\text { Additional } \\
\text { component }\end{array}$ & Aim & Cell Type & Tissue & Reference \\
\hline \multirow{6}{*}{ CNTs and GO } & CNTs & $\begin{array}{l}\text { Reinforce GelMA } \\
\text { hydrogels }\end{array}$ & $\begin{array}{c}\text { NIH-3T3 fibroblasts, } \\
\text { Human mesenchymal } \\
\text { stem cells } \\
\end{array}$ & $\begin{array}{l}\text { Connective } \\
\text { tissue }\end{array}$ & [70] \\
\hline & CNTs & $\begin{array}{l}\text { Reinforce and enhance } \\
\text { electric conductivity }\end{array}$ & $\begin{array}{c}\text { Neonatal rat ventricular } \\
\text { cardiomyocytes }\end{array}$ & Cardiac & [87] \\
\hline & CNTs & $\begin{array}{c}\text { Tune electrical and } \\
\text { mechanical properties }\end{array}$ & C2C12 myoblasts & Cardiac & [73] \\
\hline & $\begin{array}{c}\text { Aligned } \\
\text { multi-walled CNTs }\end{array}$ & $\begin{array}{l}\text { Reinforce and enhance } \\
\text { electric conductivity }\end{array}$ & $\mathrm{C} 2 \mathrm{C} 12$ muscle cells & Muscle & {$[72]$} \\
\hline & GO and MeGO & $\begin{array}{l}\text { Reinforce GelMA without } \\
\text { affecting its rigidity }\end{array}$ & NIH-3T3 fibroblasts & $\begin{array}{c}\text { Connective } \\
\text { tissue }\end{array}$ & {$[74]$} \\
\hline & GO & $\begin{array}{l}\text { Controlled gene delivery } \\
\text { vectors in terms of rate and } \\
\text { location }\end{array}$ & H9c2, HUVEC & Cardiac & {$[75]$} \\
\hline \multirow{5}{*}{ Inorganic } & $\begin{array}{l}\text { Hydroxyapatite } \\
\text { and Titanium }\end{array}$ & Enhance osseointegration & $\begin{array}{l}\text { No experiments with } \\
\text { cells }\end{array}$ & Bone & {$[82]$} \\
\hline & Minerals & Promote osteoconductivity & $\begin{array}{l}\text { No experiments with } \\
\text { cells }\end{array}$ & Bone & [81] \\
\hline & $\begin{array}{l}\text { A6ACA, PEGDA, } \\
\text { minerals }\end{array}$ & $\begin{array}{c}\text { Induce osteogenic } \\
\text { differentiation }\end{array}$ & $\begin{array}{c}\text { Human induced } \\
\text { pluripotent stem cells }\end{array}$ & Bone & [79] \\
\hline & Gold nanoparticles & Promote osteogenesis & $\begin{array}{l}\text { Adipose-Derived Stem } \\
\text { Cells }\end{array}$ & Bone & {$[78]$} \\
\hline & Silica hydrogel & $\begin{array}{l}\text { Protect cells cultured on } \\
\text { GelMA hydrogels }\end{array}$ & Cardiac side population & Cardiac & {$[80]$} \\
\hline \multirow{8}{*}{ Biopolymers } & $\begin{array}{l}\text { Gellan gum } \\
\text { methacrylate }\end{array}$ & $\begin{array}{l}\text { Reinforce GelMA } \\
\text { hydrogels }\end{array}$ & NIH-3T3 fibroblasts & Cartilage & {$[90]$} \\
\hline & $\begin{array}{c}\text { HA and } \\
\text { chondroitin sulfate } \\
\text { methacrylate }\end{array}$ & $\begin{array}{l}\text { Enhance chondrogenesis } \\
\text { and mechanical properties }\end{array}$ & Human chondrocytes & Cartilage & {$[85]$} \\
\hline & HA methacrylate & Tune physical properties & HUVEC & Cardiovascular & {$[88]$} \\
\hline & $\begin{array}{c}\text { Dextran glycidyl } \\
\text { methacrylate }\end{array}$ & $\begin{array}{c}\text { Tune mechanical } \\
\text { properties }\end{array}$ & NIH-3T3 fibroblasts & $\begin{array}{c}\text { Connective } \\
\text { tissue }\end{array}$ & {$[86]$} \\
\hline & Silk fibroin & $\begin{array}{c}\text { Tune mechanical } \\
\text { properties }\end{array}$ & NIH-3T3 fibroblasts & $\begin{array}{c}\text { Connective } \\
\text { tissue }\end{array}$ & {$[31]$} \\
\hline & PMHMGCL/PCL & $\begin{array}{c}\text { Reinforce GelMA } \\
\text { hydrogels }\end{array}$ & Healthy human cartilage & Cartilage & [99] \\
\hline & $\begin{array}{c}\text { PEG and } \\
\text { hyaluronic acid }\end{array}$ & $\begin{array}{c}\text { Regulate cell response for } \\
\text { chondrogenesis }\end{array}$ & Dental pulp stem cells & Cartilage & [97] \\
\hline & $\begin{array}{l}\text { Methacrylate and } \\
\text { lysine } \\
\text { functionalized } \\
\text { dextran }\end{array}$ & $\begin{array}{l}\text { Mimic structure and } \\
\text { function ECM by } \\
\text { combining polysaccharide } \\
\text { and protein components in } \\
\text { the hydrogel }\end{array}$ & $\begin{array}{l}\text { Human umbilical artery } \\
\text { smooth muscle cells }\end{array}$ & Vascular & [19] \\
\hline \multirow{5}{*}{$\begin{array}{l}\text { Synthetic } \\
\text { Polymers }\end{array}$} & PEG & $\begin{array}{l}\text { Tune mechanical and } \\
\text { biological properties }\end{array}$ & NIH-3T3 fibroblasts & $\begin{array}{c}\text { Connective } \\
\text { tissue }\end{array}$ & [7] \\
\hline & PLEOF & $\begin{array}{c}\text { Enhance mechanical } \\
\text { properties and degradation }\end{array}$ & Human osteoblast cells & Bone & [96] \\
\hline & Polyacrylamide & $\begin{array}{l}\text { Reinforce GelMA } \\
\text { hydrogels }\end{array}$ & L-929 cells & $\begin{array}{l}\text { Connective } \\
\text { tissue }\end{array}$ & [17] \\
\hline & PEG & $\begin{array}{c}\text { Offer to cells different } \\
\text { micro-environments to } \\
\text { induce controlled polarity } \\
\text { in embryoid bodies }\end{array}$ & $\begin{array}{l}\text { Embryoid bodies made } \\
\text { from mouse embryonic } \\
\text { stem cells }\end{array}$ & Cardiovascular & [47] \\
\hline & PEG & $\begin{array}{l}\text { Reinforce GelMA } \\
\text { hydrogels }\end{array}$ & $\begin{array}{l}\text { Endothelial cells (EA. } \\
\text { Hy926) }\end{array}$ & $\begin{array}{c}\text { Connective } \\
\text { tissue }\end{array}$ & [94] \\
\hline
\end{tabular}

